\title{
Condensate growth in trapped Bose gases
}

\author{
M. J. Bijlsma, E. Zaremba, ${ }^{*}$ and H. T. C. Stoof \\ Institute for Theoretical Physics, University of Utrecht, Princetonplein 5, 3584 CC Utrecht, The Netherlands
}

(Received 21 January 2000; published 15 November 2000)

\begin{abstract}
We study the dynamics of condensate formation in an inhomogeneous trapped Bose gas with a positive interatomic scattering length. We take into account both the nonequilibrium kinetics of the thermal cloud and the Hartree-Fock mean-field effects in the condensed and the noncondensed parts of the gas. Our growth equations are solved numerically by assuming that the thermal component behaves ergodically and that the condensate, treated within the Thomas-Fermi approximation, grows adiabatically. Our simulations are in good qualitative agreement with experiment, however important discrepancies concerning details of the growth behavior remain.
\end{abstract}

PACS number(s): 03.75.Fi, 32.80.Pj, 05.30.Jp

\section{INTRODUCTION}

The discovery of Bose-Einstein condensation (BEC) in trapped atomic vapors of ${ }^{87} \mathrm{Rb}[1],{ }^{7} \mathrm{Li}[2]$, and ${ }^{23} \mathrm{Na}[3]$ has initiated a period of intense experimental and theoretical activity. A great deal of information is now available about the equilibrium properties of these novel systems [4], but much remains to be understood about their nonequilibrium behavior. One of the most basic aspects concerns the nonequilibrium growth of the condensate that occurs in the process of cooling a nondegenerate trapped Bose gas to a final temperature below the BEC transition. This important problem was addressed even before the first observation of BEC in trapped atomic gases [5-7], and has interesting implications for the general problem of second-order phase transitions, from superfluidity in liquid ${ }^{4} \mathrm{He}[8]$ to problems in cosmology [9].

Until now, the most detailed study of condensate formation was carried out using a gas of ${ }^{23} \mathrm{Na}$ atoms confined within a highly anisotropic cigar-shaped trap [10]. In these experiments, the sodium atoms were evaporatively cooled to a temperature just above the critical temperature and subsequently quenched by applying a rapid RF sweep. The latter step removes all, or at least a large fraction, of the atoms above a certain energy, after which the Bose gas relaxes to a new equilibrium state below the critical temperature. The growth of the condensate during the equilibration process was monitored using a nondestructive imaging technique that provided a direct measure of the size of the condensate as a function of time. In this way, the characteristic time scale for the growth of the condensate could be determined, and for the particular system studied, was found to be of the order of $100 \mathrm{~ms}$.

A theoretical description of these experiments requires a theory that can account for the coupled nonequilibrium dynamics of both the noncondensed and condensed components of a trapped Bose gas, and includes in particular the collisional processes that transfer atoms between the two components. Thus far, several such theories have been de-

\footnotetext{
*Permanent address: Department of Physics, Queen's University, Kingston, Ontario, Canada K7L 3N6.
}

veloped, which roughly speaking fall into two categories. One class of theories focuses on describing the dynamics of the average value of the order parameter for BEC, i.e., the condensate wave function, whereas the other incorporates also the fluctuations around this mean value. The latter of course, becomes important when the fluctuations are large compared to the mean value, i.e., close to the critical temperature. This is analogous to the situation in laser theory [11].

A theory that describes both the average value for the order parameter as well its fluctuations can be obtained in two, essentially equivalent ways. First, one can start from a master equation for the many-body density matrix and derive an equation of motion for the one-particle density matrix by means of a perturbative treatment of the interactions. This was the route followed by Gardiner and Zoller [12], in a series of papers. Second, one can use field-theoretic methods to obtain a nonperturbative Fokker-Planck equation that describes the nonequilibrium dynamics of the gas. This was the formulation developed by Stoof [13]. These two approaches in principle yield a description of the nonequilibrium dynamics that is capable of obtaining the complete probability distribution for the order parameter.

Alternatively, a theory describing the dynamics of the mean-field value for the macroscopic wave function can also be obtained in a straightforward decoupling approach, which has been implemented by Kirkpatrick and Dorfmann [14], Proukakis et al. [15], Walser et al. [16], and in the most detail for the trapped case by Zaremba, Nikuni, and Griffin [17]. In this approach, one assumes the order parameter to be nonzero at all temperatures, and decouples the hierarchy of equations of motion that exists for the correlation functions of the second-quantized field operators. Thus one again obtains a perturbative expansion for these equations of motion.

The first quantitative calculations of condensate growth for trapped Bose gases were carried out by Gardiner et al. [18], and although good qualitative agreement with experiment was found, a number of quantitative discrepancies remained. For example, the reported experimental growth rates were up to a factor 30 larger than the initial theoretical results, and had a temperature dependence opposite to that predicted [10]. By removing some simplifying approximations in subsequent calculations, the theoretical results were im- 
proved, but discrepancies of up to a factor of 3 still remained in some cases. From a purely theoretical point of view, one can attribute some of these discrepancies to the approximations made in the calculations. First, the dynamics of the noncondensate was to a large extent neglected. Although the time evolution of the occupancy of low-lying states was included in the simulations, the high-energy states were represented by an equilibrium particle reservoir having a fixed chemical potential. This latter assumption is inconsistent with the nonequilibrium initial state established by the experimental quench procedure. Second, the effect of the mean field of the condensate on the noncondensate was included rather crudely by a linear rescaling of the low-energy density of states of the noncondensed atoms.

Our aim in the present paper is to improve on these calculations by taking fully into account the relaxational dynamics of the thermal, or noncondensed, component that takes place in the presence of the mean field of the condensate. We do this by starting from the above-mentioned theories describing the growth process, which provide us with a nonlinear Schrödinger equation for the condensate and a kinetic equation for the thermal component. This coupled set of equations is still difficult to deal with and a number of physically motivated approximations are made to simplify the problem. We assume that the condensate grows adiabatically, having an equilibrium spatial distribution determined by the instantaneous number of atoms in the condensate. This assumption is also made in earlier work [18]. The noncondensate is treated by solving a semiclassical Boltzmann equation $[13,14,17,19]$ in the ergodic approximation, which again has been used previously by numerous authors $[6,20-$ $22]$. These assumptions allow us to obtain numerically a detailed description of the growth of the condensate, including the effects of both the dynamics of the thermal cloud and its mean-field interaction with the condensate.

The paper is organized as follows. In Sec. II, we summarize the theory of the nonequilibrium dynamics of a trapped Bose gas as developed previously. In Sec. III, we introduce the central assumption, the ergodic approximation, that allows us to numerically solve the Boltzmann equation. In addition, we briefly discuss the adiabatic approximation for the condensate. In Sec. IV, we treat in some detail particle number and energy conservation. Sec. V introduces the ThomasFermi approximation and gives some analytical results for the density of states and other quantities of interest. The numerical solution of our kinetic equations is discussed in Sec. VI and our results for the growth of a condensate are presented. We end in Sec. VII with a discussion and an outlook.

\section{NONEQUILIBRIUM DYNAMICS}

As discussed in the previous section, the nonequilibrium dynamics of a trapped Bose gas is governed by a set of equations for the condensate and noncondensate components. These equations have been presented in various forms [18,13-17], but they all describe the coherent dynamics of the gas due to mean-field interactions, as well as the incoherent dynamics associated with atomic collisions. The equa- tions and notation we use are taken from Refs. [13,17].

The noncondensate is treated using a semiclassical Boltzmann equation for the phase-space distribution function $f(\mathbf{r}, \mathbf{p}, t)$. This semiclassical description is justified when the largest level spacing in the external trapping potential is small compared to the thermal excitation energy. Moreover, mean-field interactions are included at the level of the Hartree-Fock approximation. In this situation, the quantum kinetic equation for the thermal excitations takes the form $[13,17]$

$$
\begin{aligned}
& \frac{\partial f(\mathbf{r}, \mathbf{p}, t)}{\partial t}+\frac{\mathbf{p}}{m} \cdot \boldsymbol{\nabla} f(\mathbf{r}, \mathbf{p}, t)-\boldsymbol{\nabla} U(\mathbf{r}, t) \cdot \nabla_{\mathbf{p}} f(\mathbf{r}, \mathbf{p}, t) \\
& \quad=C_{12}[f]+C_{22}[f] .
\end{aligned}
$$

Here, the effective potential $U(\mathbf{r}, t) \equiv U_{\text {ext }}(\mathbf{r})+2 g\left[n_{c}(\mathbf{r}, t)\right.$ $+\tilde{n}(\mathbf{r}, t)]$ is the sum of the external trapping potential $U_{\text {ext }}$ and the self-consistent Hartree-Fock mean field. The latter is determined by the condensate density $n_{c}(\mathbf{r}, t)$, defined below, and the noncondensate density $\tilde{n}(\mathbf{r}, t)$ given by

$$
\tilde{n}(\mathbf{r}, t)=\int \frac{d \mathbf{p}}{(2 \pi \hbar)^{3}} f(\mathbf{r}, \mathbf{p}, t) .
$$

As usual, we treat the interactions in the $s$-wave approximation, which results in the bare interaction being replaced by a contact interaction with an effective coupling constant $g$ $=4 \pi \hbar^{2} \mathrm{a} / \mathrm{m}$ proportional to the $s$-wave scattering length $a$. The effective coupling constant is in fact equal to the twobody $T$ matrix, and to emphasize this connection, it is denoted by $T^{2 B}$ in some works [13]. The collision terms appearing in Eq. (1) are given by

$$
\begin{aligned}
C_{22}[f] \equiv & \frac{4 \pi}{\hbar} g^{2} \int \frac{d \mathbf{p}_{2}}{(2 \pi \hbar)^{3}} \int \frac{d \mathbf{p}_{3}}{(2 \pi \hbar)^{3}} \int \frac{d \mathbf{p}_{4}}{(2 \pi \hbar)^{3}}(2 \pi \hbar)^{3} \\
& \times \delta\left(\mathbf{p}+\mathbf{p}_{2}-\mathbf{p}_{3}-\mathbf{p}_{4}\right) \delta\left(E+E_{2}-E_{3}-E_{4}\right)[(1+f) \\
& \left.\times\left(1+f_{2}\right) f_{3} f_{4}-f f_{2}\left(1+f_{3}\right)\left(1+f_{4}\right)\right], \\
C_{12}[f] \equiv & \frac{4 \pi}{\hbar} g^{2} n_{c} \int \frac{d \mathbf{p}_{2}}{(2 \pi \hbar)^{3}} \int \frac{d \mathbf{p}_{3}}{(2 \pi \hbar)^{3}} \int \frac{d \mathbf{p}_{4}}{(2 \pi \hbar)^{3}} \\
& \times(2 \pi \hbar)^{3} \delta\left(m \mathbf{v}_{c}+\mathbf{p}_{2}-\mathbf{p}_{3}-\mathbf{p}_{4}\right) \delta\left(E_{c}+E_{2}-E_{3}\right. \\
& \left.-E_{4}\right)(2 \pi \hbar)^{3}\left[\delta\left(\mathbf{p}-\mathbf{p}_{2}\right)-\delta\left(\mathbf{p}-\mathbf{p}_{3}\right)-\delta\left(\mathbf{p}-\mathbf{p}_{4}\right)\right] \\
& \times\left[\left(1+f_{2}\right) f_{3} f_{4}-f_{2}\left(1+f_{3}\right)\left(1+f_{4}\right)\right],
\end{aligned}
$$

with $f \equiv f(\mathbf{r}, \mathbf{p}, t)$, and $f_{i} \equiv f\left(\mathbf{r}, \mathbf{p}_{i}, t\right)$. We note that Eq. (4) takes into account the fact that a condensate atom locally has an energy

$$
E_{c}(\mathbf{r}, t)=\mu_{c}(\mathbf{r}, t)+\frac{1}{2} m \mathbf{v}_{c}^{2}(\mathbf{r}, t)
$$


a momentum $m \mathbf{v}_{c}(\mathbf{r}, t)$, and a chemical potential $\mu_{c}(\mathbf{r}, t)$. These quantities are defined explicitly below. In addition, the energy of a noncondensate atom in the Hartee-Fock approximation is

$$
E(\mathbf{r}, \mathbf{p}, t)=\frac{\mathbf{p}^{2}}{2 m}+U(\mathbf{r}, t) .
$$

The energy variables $E_{i}$ appearing in Eqs. (3) and (4) are defined as $E_{i}=E\left(\mathbf{r}, \mathbf{p}_{i}, t\right)$.

In contrast to the thermal cloud, the dynamics of the condensate is determined by a time-dependent dissipative nonlinear Schrödinger equation $[13,17]$

$$
\begin{aligned}
i \hbar \frac{\partial \Psi(\mathbf{r}, t)}{\partial t}= & \left\{-\frac{\hbar^{2} \nabla^{2}}{2 m}+U_{\mathrm{ext}}(\mathbf{r})+g\left[2 \tilde{n}(\mathbf{r}, t)+n_{c}(\mathbf{r}, t)\right]\right. \\
& -i R(\mathbf{r}, t)\} \Psi(\mathbf{r}, t),
\end{aligned}
$$

where the dissipative term, i.e., $R(\mathbf{r}, t)$, is given by

$$
\begin{aligned}
R \equiv & \frac{\hbar}{2 n_{c}} \int \frac{d \mathbf{p}}{(2 \pi \hbar)^{3}} C_{12}[f] \\
= & \frac{g^{2}}{(2 \pi)^{5} \hbar^{6}} \int \prod_{i=1,4} d \mathbf{p}_{i} \delta\left(m \mathbf{v}_{c}+\mathbf{p}_{2}-\mathbf{p}_{3}-\mathbf{p}_{4}\right) \delta\left(E_{c}+E_{2}\right. \\
& \left.-E_{3}-E_{4}\right)\left[\delta\left(\mathbf{p}_{1}-\mathbf{p}_{2}\right)-\delta\left(\mathbf{p}_{1}-\mathbf{p}_{3}\right)-\delta\left(\mathbf{p}_{1}-\mathbf{p}_{4}\right)\right] \\
& \times\left[\left(1+f_{2}\right) f_{3} f_{4}-f_{2}\left(1+f_{3}\right)\left(1+f_{4}\right)\right] .
\end{aligned}
$$

The appearance of this dissipative term in Eq. (7) is a consequence of the collisional processes, described by $C_{12}$, which have the effect of transfering particles between the condensate and noncondensate. The dissipative term is needed in order to ensure overall particle-number conservation of the entire system. At a more fundamental level, the condensate wave function is determined by taking the expectation value of a Bose field with respect to a probability distribution that satisfies the Fokker-Planck equation mentioned previously [13]. The Langevin equation one derives within this formulation has the form of a dissipative nonlinear Schrödinger equation with a noise term. It only reduces to Eq. (7) in a mean-field approximation. This points to the need for exercising care in interpreting the order parameter occurring in the nonlinear Schrödinger equation as the condensate wave function. Due to the underlying $U(1)$ gauge invariance associated with strict particle-number conservation, the expectation value is in fact always equal to zero as a result of the diffusion of the global phase of the condensate wave function. In first instance this effect can be neglected and we are then effectively treating the system as if the $U(1)$ gauge invariance is explicitly broken.

It is convenient to rewrite Eq. (7) in terms of amplitude and phase variables defined by $\Psi(\mathbf{r}, t)$ $=\sqrt{n_{c}(\mathbf{r}, t)} \exp [i \theta(\mathbf{r}, t)]$. Substituting this form of the wave function into Eq. (7), we obtain

$$
\frac{\partial n_{c}(\mathbf{r}, t)}{\partial t}+\boldsymbol{\nabla}\left[\mathbf{v}_{c}(\mathbf{r}, t) n_{c}(\mathbf{r}, t)\right]=-\frac{2}{\hbar} R(\mathbf{r}, t) n_{c}(\mathbf{r}, t),
$$

and

$$
m \frac{\partial \mathbf{v}_{c}(\mathbf{r}, t)}{\partial t}+\boldsymbol{\nabla}\left[\mu_{c}(\mathbf{r}, t)+\frac{m \mathbf{v}_{c}(\mathbf{r}, t)^{2}}{2}\right]=\mathbf{0} .
$$

Here, we have defined the local chemical potential and superfluid velocity by

$$
\mu_{c}(\mathbf{r}, t)=U_{\text {ext }}(\mathbf{r})+g\left[n_{c}(\mathbf{r}, t)+2 \tilde{n}(\mathbf{r}, t)\right]-\frac{\hbar^{2}}{2 m} \frac{\nabla^{2} \sqrt{n_{c}(\mathbf{r}, t)}}{\sqrt{n_{c}(\mathbf{r}, t)}}
$$

and

$$
\mathbf{v}_{c}(\mathbf{r}, t)=\frac{\hbar}{m} \boldsymbol{\nabla} \theta(\mathbf{r}, t),
$$

respectively. It can easily be shown that this set of equations for the condensate and thermal cloud is consistent with the conservation of the total number of particles in the system [17].

\section{ERGODIC APPROXIMATION}

Our main objective in this paper is to apply the kinetic theory formulated above to the problem of condensate formation. In order to make progress, we introduce a number of additional approximations. The first and most essential, is the assumption of ergodicity $[6,20-22]$ that has been widely used in the literature on kinetic theory. This assumes that equilibration of atoms within one energy level occurs on a much shorter time scale than equilibration of atoms between different energy levels. With this assumption, all points in phase space having the same energy are equally probable, and the distribution function therefore only depends on the phase-space variables through the energy variable $E(\mathbf{r}, \mathbf{p}, t)$, i.e., $f(\mathbf{r}, \mathbf{p}, t) \equiv g(E(\mathbf{r}, \mathbf{p}, t), t)$. In equilibrium this is certainly correct, but the assumption requires justification for any particular nonequilibrium application. Unfortunately, we are not aware of any explicit checks that have been made that might indicate that the assumption is correct for the situations we wish to consider. Nevertheless, it appears to be physically reasonable that for quantities that vary on a time scale of the order of several collision times, the approximation is sufficiently accurate.

The ergodic approximation allows us to derive a simplified kinetic equation for the energy distribution function $g(\epsilon, t)$. This is accomplished by means of the relation

$$
\rho(\epsilon, t) g(\epsilon, t) \equiv \int \frac{d \mathbf{r} d \mathbf{p}}{(2 \pi \hbar)^{3}} \delta(\epsilon-E(\mathbf{r}, \mathbf{p}, t)) f(\mathbf{r}, \mathbf{p}, t),
$$

which shows that the phase-space projection defined on the right-hand side yields the product of $g(\epsilon, t)$ and the density of states 


$$
\begin{aligned}
\rho(\epsilon, t) & =\int \frac{d \mathbf{r} d \mathbf{p}}{(2 \pi \hbar)^{3}} \delta(\epsilon-E(\mathbf{r}, \mathbf{p}, t)) \\
& =\frac{m^{3 / 2}}{\sqrt{2} \pi^{2} \hbar^{3}} \int_{U \leqslant \epsilon} d \mathbf{r} \sqrt{\epsilon-U(\mathbf{r}, t)} .
\end{aligned}
$$

We note that the density of states is defined on the variable energy range $U_{\min }(t) \leqslant \epsilon<\infty$ where $U_{\min }(t)$ is the minimum value of $U(\mathbf{r}, t)$ at time $t$. The time dependence of the density of states is one of the aspects distinguishing the present development from previous work [20,21].

We now apply the phase-space projection to the kinetic equation in Eq. (1). As a result of this operation, the streaming terms in the Boltzmann equation, i.e., the second and third terms on the left-hand side of Eq. (1), cancel each other. Only the projection of the time-derivative term survives. This results in

$$
\begin{gathered}
\int \frac{d \mathbf{r} d \mathbf{p}}{(2 \pi \hbar)^{3}} \delta(\epsilon-E(\mathbf{r}, \mathbf{p}, t)) \frac{\partial f(\mathbf{r}, \mathbf{p}, t)}{\partial t} \\
=\rho(\epsilon, t) \frac{\partial g(\epsilon, t)}{\partial t}+\rho_{\mathrm{w}}(\epsilon, t) \frac{\partial g(\boldsymbol{\epsilon}, t)}{\partial \epsilon} .
\end{gathered}
$$

Here, we have introduced a weighted density of states

$$
\begin{aligned}
\rho_{\mathrm{w}}(\boldsymbol{\epsilon}, t) & =\int \frac{d \mathbf{r} d \mathbf{p}}{(2 \pi \hbar)^{3}} \delta(\epsilon-E(\mathbf{r}, \mathbf{p}, t)) \frac{\partial U(\mathbf{r}, t)}{\partial t} \\
& =\frac{m^{3 / 2}}{\sqrt{2} \pi^{2} \hbar^{3}} \int_{U \leqslant \epsilon} d \mathbf{r} \sqrt{\epsilon-U(\mathbf{r}, t)} \frac{\partial U(\mathbf{r}, t)}{\partial t}
\end{aligned}
$$

This quantity depends explicitly on the time derivative of the noncondensate potential that in turn is determined by the time derivatives of both the condensate and noncondensate densities. Some formal details regarding its evaluation are given in the Appendix.

Noting that

$$
\frac{\partial \rho(\epsilon, t)}{\partial t}=-\frac{\partial \rho_{\mathrm{w}}(\epsilon, t)}{\partial \epsilon}
$$

Eq. (15) can be written as

$$
\int \frac{d \mathbf{r} d \mathbf{p}}{(2 \pi \hbar)^{3}} \delta(\epsilon-E(\mathbf{r}, \mathbf{p}, t)) \frac{\partial f(\mathbf{r}, \mathbf{p}, t)}{\partial t}=\frac{\partial}{\partial t}(\rho g)+\frac{\partial}{\partial \epsilon}\left(\rho_{\mathrm{w}} g\right) .
$$

We thus arrive at the projected kinetic equation

$$
\frac{\partial}{\partial t}(\rho g)+\frac{\partial}{\partial \epsilon}\left(\rho_{\mathrm{w}} g\right)=I_{12}+I_{22}
$$

where the phase-space projections of the collision integrals are defined as

$$
\begin{aligned}
& I_{12}(\epsilon, t) \equiv \int \frac{d \mathbf{r} d \mathbf{p}}{(2 \pi \hbar)^{3}} \delta(\epsilon-E(\mathbf{r}, \mathbf{p}, t)) C_{12}[f] \\
& I_{22}(\epsilon, t) \equiv \int \frac{d \mathbf{r} d \mathbf{p}}{(2 \pi \hbar)^{3}} \delta(\epsilon-E(\mathbf{r}, \mathbf{p}, t)) C_{22}[f] .
\end{aligned}
$$

The result in Eq. (19) is the kinetic equation that we solve numerically.

We now derive in some detail explicit expressions for the collision integrals in Eq. (20). Although an expression for $I_{22}$ was given in earlier work [20], we present here an alternative derivation that can also be adapted to the case of the $I_{12}$ collision integral. For the $I_{22}$ collision integral we have

$$
\begin{aligned}
I_{22}\left(\boldsymbol{\epsilon}_{1}, t\right)= & \frac{4 \pi g^{2}}{(2 \pi)^{9} \hbar^{10}} \int d \epsilon_{2} \int d \boldsymbol{\epsilon}_{3} \int d \boldsymbol{\epsilon}_{4} \delta\left(\boldsymbol{\epsilon}_{1}+\boldsymbol{\epsilon}_{2}-\boldsymbol{\epsilon}_{3}\right. \\
& \left.-\boldsymbol{\epsilon}_{4}\right)\left[\left(1+g_{1}\right)\left(1+g_{2}\right) g_{3} g_{4}-g_{1} g_{2}\left(1+g_{3}\right)\right. \\
& \left.\times\left(1+g_{4}\right)\right] \int d \mathbf{r}\left(\prod_{i=1,4} \int d \mathbf{p}_{i}\right) \delta\left(\mathbf{p}_{1}+\mathbf{p}_{2}\right. \\
& \left.-\mathbf{p}_{3}-\mathbf{p}_{4}\right) \delta\left(\boldsymbol{\epsilon}_{1}-E_{1}\right) \delta\left(\boldsymbol{\epsilon}_{2}-E_{2}\right) \delta\left(\boldsymbol{\epsilon}_{3}-E_{3}\right) \\
& \times \delta\left(\epsilon_{4}-E_{4}\right),
\end{aligned}
$$

where we have introduced the shorthand notation $g_{i}$ $=g\left(\epsilon_{i}, t\right)$. We consider first the momentum integrals in Eq. (21) which, with the replacement $\mathbf{p}_{3} \rightarrow-\mathbf{p}_{3}$ and $\mathbf{p}_{4} \rightarrow-\mathbf{p}_{4}$, can be written as

$$
\begin{aligned}
J_{22} \equiv & \int d \mathbf{p}_{1} \int d \mathbf{p}_{2} \int d \mathbf{p}_{3} \int d \mathbf{p}_{4} \delta\left(\mathbf{p}_{1}+\mathbf{p}_{2}+\mathbf{p}_{3}+\mathbf{p}_{4}\right) \\
& \times \delta\left(\epsilon_{1}-E_{1}\right) \delta\left(\epsilon_{2}-E_{2}\right) \delta\left(\boldsymbol{\epsilon}_{3}-E_{3}\right) \delta\left(\boldsymbol{\epsilon}_{4}-E_{4}\right) \\
= & \int \frac{d \boldsymbol{\xi}}{(2 \pi)^{3}} \prod_{i=1}^{4} \int d \mathbf{p}_{i} e^{i \boldsymbol{\xi} \cdot \mathbf{p}_{i}} \delta\left(\boldsymbol{\epsilon}_{i}-E_{i}\right)
\end{aligned}
$$

In obtaining this expression, we have introduced a Fourier representation of the momentum-conserving delta function. Performing the integrals in Eq. (22) with respect to the momentum variables, we obtain

$$
\begin{aligned}
J_{22}= & (4 \pi m)^{4}\left(\prod_{i=1}^{4} \theta\left(\epsilon_{i}-U\right)\right) \\
& \times \int \frac{d \boldsymbol{\xi}}{(2 \pi)^{3}} \frac{\sin \xi p_{1} \sin \xi p_{2} \sin \xi p_{3} \sin \xi p_{4}}{\xi^{4}},
\end{aligned}
$$

where now it is understood that $p_{i}=\sqrt{2 m\left(\epsilon_{i}-U\right)}$. The product of theta functions can be replaced by $\theta\left(\epsilon_{\min }-U\right)$, with $\epsilon_{\min }$ the minimum value of the four energy variables. Performing the remaining integral with respect to the $\boldsymbol{\xi}$ variable, we find 


$$
\begin{aligned}
J_{22}= & (2 \pi)^{3} m^{4} \theta\left(\epsilon_{\min }-U\right)\left[\left|p_{1}-p_{2}+p_{3}+p_{4}\right|\right. \\
& -\left|p_{1}-p_{2}+p_{3}-p_{4}\right|+\left|p_{1}-p_{2}-p_{3}-p_{4}\right| \\
& -\left|p_{1}-p_{2}-p_{3}+p_{4}\right|+\left|p_{1}+p_{2}+p_{3}-p_{4}\right| \\
& -\left|p_{1}+p_{2}-p_{3}-p_{4}\right|+\left|p_{1}+p_{2}-p_{3}+p_{4}\right| \\
& \left.-\left|p_{1}+p_{2}+p_{3}+p_{4}\right|\right] .
\end{aligned}
$$

This expression is valid for arbitrary values of the momenta but simplifies when energy conservation is taken into account. Since the energy-conserving delta function $\delta\left(\epsilon_{1}+\epsilon_{2}\right.$ $\left.-\epsilon_{3}-\epsilon_{4}\right)$ in Eq. (21) imposes the constraint $p_{1}^{2}+p_{2}^{2}=p_{3}^{2}$ $+p_{4}^{2}$, Eq. (24) can be reduced to

$$
J_{22}=4(2 \pi)^{3} m^{4} \theta\left(\epsilon_{\min }-U\right) \sqrt{2 m\left(\epsilon_{\min }-U\right)} .
$$

Substituting this expression for $J_{22}$ into Eq. (21), we finally obtain

$$
\begin{aligned}
I_{22}\left(\epsilon_{1}, t\right)= & \frac{m^{3} g^{2}}{2 \pi^{3} \hbar^{7}} \int d \epsilon_{2} \int d \epsilon_{3} \int d \epsilon_{4} \rho\left(\epsilon_{\min }\right) \\
& \times \delta\left(\epsilon_{1}+\epsilon_{2}-\epsilon_{3}-\epsilon_{4}\right)\left[\left(1+g_{1}\right)\left(1+g_{2}\right) g_{3} g_{4}\right. \\
& \left.-g_{1} g_{2}\left(1+g_{3}\right)\left(1+g_{4}\right)\right],
\end{aligned}
$$

where we have used the definition of the density of states in Eq. (14). This is precisely the result obtained by Snoke and Wolfe [6] and Luiten, Reynolds, and Walraven [20], using a different method. We note that if all energies are expressed in units of $\hbar \bar{\omega}=\hbar\left(\omega_{1} \omega_{2} \omega_{3}\right)^{1 / 3}$, the $I_{22}$ integral has an overall factor of $(a / l)^{2} \bar{\omega}$, where $l=\sqrt{\hbar / m \bar{\omega}}$ is the average harmonic-oscillator length. This factor defines a characteristic time that can be used as the time unit in the simulations.

The $I_{12}$ collision integral can be dealt with in a similar way if the superfluid velocity $\mathbf{v}_{c}$ in Eq. (4) is set to zero. The validity of this approximation follows from our assumption that the condensate grows adiabatically. The magnitude of the superfluid velocity $\mathbf{v}_{c}$ is then typically of the order of $\dot{R}(t)$, where $R(t)$ is the radius of the condensate. This velocity is small compared to the characteristic velocities $\mathbf{p} / m$ $\approx \sqrt{2 k_{B} T / m}$ of the thermal atoms participating in a collision, which justifies the neglect of $m \mathbf{v}_{c}$ in Eq. (4). The expression for $I_{12}$ then reads

$$
\begin{aligned}
I_{12}\left(\epsilon_{1}, t\right)= & \frac{4 \pi g^{2}}{(2 \pi)^{6} \hbar^{7}} \int d \mathbf{r} n_{c}(\mathbf{r}, t) \\
& \times \int d \epsilon_{2} \int d \epsilon_{3} \int d \epsilon_{4} \delta\left(E_{c}(\mathbf{r}, t)+\epsilon_{2}-\epsilon_{3}-\epsilon_{4}\right) \\
& \times\left[\delta\left(\epsilon_{1}-\epsilon_{2}\right)-\delta\left(\epsilon_{1}-\epsilon_{3}\right)-\delta\left(\epsilon_{1}-\epsilon_{4}\right)\right] \\
& \times\left[\left(1+g_{2}\right) g_{3} g_{4}-g_{2}\left(1+g_{3}\right)\left(1+g_{4}\right)\right] \\
& \times \int d \mathbf{p}_{2} \int d \mathbf{p}_{3} \int d \mathbf{p}_{4} \delta\left(\mathbf{p}_{2}-\mathbf{p}_{3}-\mathbf{p}_{4}\right) \\
& \times \delta\left(\epsilon_{2}-E_{2}\right) \delta\left(\epsilon_{3}-E_{3}\right) \delta\left(\epsilon_{4}-E_{4}\right) .
\end{aligned}
$$

If we now define $J_{12}$ analogously to $J_{22}$, we have

$$
\begin{aligned}
J_{12} \equiv & \int d \mathbf{p}_{2} \int d \mathbf{p}_{3} \int d \mathbf{p}_{4} \delta\left(\mathbf{p}_{2}+\mathbf{p}_{3}+\mathbf{p}_{4}\right) \\
& \times \delta\left(\epsilon_{2}-E_{2}\right) \delta\left(\epsilon_{3}-E_{3}\right) \delta\left(\epsilon_{4}-E_{4}\right) \\
= & \int \frac{d \boldsymbol{\xi}}{(2 \pi)^{3}} \prod_{i=2}^{4} \int d \mathbf{p}_{i} e^{i \xi \cdot \mathbf{p}_{i}} \delta\left(\epsilon_{i}-E_{i}\right) \\
= & (4 \pi m)^{3} \theta\left(\epsilon_{\min }-U\right) \int \frac{d \boldsymbol{\xi}}{(2 \pi)^{3}} \frac{\sin \xi p_{2} \sin \xi p_{3} \sin \xi p_{4}}{\xi^{3}} \\
= & 8 \pi^{2} m^{3} \theta\left(\epsilon_{\min }-U\right) S\left(p_{2}, p_{3}, p_{4}\right),
\end{aligned}
$$

where $\epsilon_{\min }$ is the minimum value of $\epsilon_{2}, \epsilon_{3}$, and $\epsilon_{4}$, and

$$
\begin{aligned}
S\left(p_{2}, p_{3}, p_{4}\right) \equiv & \frac{1}{2}\left[\operatorname{sgn}\left(p_{2}+p_{3}-p_{4}\right)+\operatorname{sgn}\left(p_{2}-p_{3}+p_{4}\right)\right. \\
& \left.-\operatorname{sgn}\left(p_{2}+p_{3}+p_{4}\right)-\operatorname{sgn}\left(p_{2}-p_{3}-p_{4}\right)\right] .
\end{aligned}
$$

Note that this is a Boolean function that takes on values of 0 and 1 only. Inserting the expression for $J_{12}$ into Eq. (27), we finally obtain for the $I_{12}$ collision integral the result

$$
\begin{aligned}
I_{12}\left(\epsilon_{1}, t\right)= & \frac{m^{3} g^{2}}{2 \pi^{3} \hbar^{7}} \int d \epsilon_{2} \int d \epsilon_{3} \int d \epsilon_{4}\left[\delta\left(\epsilon_{1}-\epsilon_{2}\right)\right. \\
& \left.-\delta\left(\epsilon_{1}-\epsilon_{3}\right)-\delta\left(\epsilon_{1}-\epsilon_{4}\right)\right]\left[\left(1+g_{2}\right) g_{3} g_{4}\right. \\
& \left.-g_{2}\left(1+g_{3}\right)\left(1+g_{4}\right)\right] \int_{U \leqslant \epsilon_{\min }} d \mathbf{r} n_{c}(\mathbf{r}, t) \\
& \times S\left(p_{2}, p_{3}, p_{4}\right) \delta\left(E_{c}(\mathbf{r}, t)+\epsilon_{2}-\epsilon_{3}-\epsilon_{4}\right) .
\end{aligned}
$$

A comparison of this expression with $I_{22}$ in Eq. (26) shows that the remaining spatial integral acts as an effective density of states for scattering into the condensate. It can be evaluated analytically in the Thomas-Fermi approximation for the condensate, as shown in Sec. V. The kinetic equation in Eq. (19) and the projected collision integrals in Eqs. (26) and (30) are the main results of this section.

Before closing this section, we point out one difficulty encountered when a numerical solution of Eq. (19) is attempted. As discussed following Eq. (14), the time dependence of the mean-field potential $U(\mathbf{r}, t)$ implies that the density of states in Eq. (14), and hence the energy distribution function $g(\epsilon, t)$, are defined on a variable energy range. To eliminate this variation, it is convenient to introduce the shifted energy variable

$$
\bar{\epsilon} \equiv \epsilon-U_{\min }(t),
$$

which leads to a fixed energy range $0 \leqslant \bar{\epsilon}<\infty$. The density of states in terms of this new energy variable is given by $\rho(\epsilon, t)=\rho\left(\bar{\epsilon}+U_{\min }, t\right) \equiv \bar{\rho}(\bar{\epsilon}, t)$. With $\bar{\epsilon}$ and $t$ as independent variables, the kinetic equation in Eq. (19) can be rewritten as 


$$
\frac{\partial}{\partial t}(\bar{\rho} \bar{g})+\frac{\partial}{\partial \bar{\epsilon}}\left(\bar{\rho}_{\mathrm{w}} \bar{g}\right)=\bar{I}_{12}+\bar{I}_{22} .
$$

Here, both $\bar{\rho}(\bar{\epsilon}, t)$ and $\bar{\rho}_{\mathrm{w}}(\bar{\epsilon}, t)$ are defined by making the replacement

$$
U(\mathbf{r}, t) \rightarrow U(\mathbf{r}, t)-U_{\min }(t) \equiv \bar{U}(\mathbf{r}, t)
$$

in Eqs. (14) and (16). Similarly, from the definition of the collision integrals in Eqs. (3) and (4), it can also be seen that the change of energy variable leads to the replacement of $U(\mathbf{r}, t)$ by $\bar{U}(\mathbf{r}, t)$ in this case as well. Thus, the final kinetic equation in terms of the $\bar{\epsilon}$ variable is unchanged in form from the original equation. We will henceforth drop the overbar on the functions defined in terms of $\bar{\epsilon}$, with the understanding that the shifted potential $\bar{U}(\mathbf{r}, t)$ is to be used wherever the potential appears in the original expressions. The possibility of using a fixed energy range in the solution of the kinetic equation simplifies the numerical calculations considerably.

\section{COLLISIONAL INVARIANTS}

In this section, we explicitly consider two important quantities that should be conserved as the Bose gas condenses and equilibrates, namely, the total number of particles and the total energy of the trapped Bose gas. Together, they determine the final equilibrium state of the Bose-condensed gas, i.e., the number of particles in the condensate, its chemical potential, and the temperature of the vapor.

\section{A. Particle-number conservation}

The time rate of change of the total number of particles consists of the time rate of change of the number of condensed particles plus the time rate of change of the number of noncondensed particles. Because the number of noncondensed particles is given by $\widetilde{N}(t)=(2 \pi \hbar)^{-3} \int d \mathbf{r} d \mathbf{p} f(\mathbf{p}, \mathbf{r}, t)$ $=\int d \epsilon \rho(\epsilon) g(\epsilon)$, the time rate of change of $\widetilde{N}(t)$ can be found by integrating Eq. (19) over energy. We thus find that

$$
\frac{\partial \tilde{N}(t)}{\partial t}=\int d \epsilon I_{12}(\epsilon, t),
$$

where it is easily checked from Eq. (26) that

$$
\int d \epsilon I_{22}(\epsilon, t)=0 \text {. }
$$

Note that we have assumed here that $\lim _{\epsilon \rightarrow U_{\min }} \rho_{\mathrm{w}}(\epsilon) g(\epsilon)$ $=0$. A finite limiting value can arise if $g(\epsilon)$ approaches an equilibrium Bose distribution with a chemical potential $\mu$ $=U_{\min }$ at long times, together with a weighted density of states that depends linearly on $\epsilon-U_{\min }$ for energies close to $U_{\text {min }}$. However, at any finite time in the growth process, it is safe to use the zero limiting value. This is always the case when the equilibrium chemical potential lies below $U_{\min }$.
To get the time rate of change of the total number of condensate particles, we integrate the continuity equation, Eq. (9), over space to find

$$
\frac{\partial N_{c}}{\partial t}=-\frac{2}{\hbar} \int d \mathbf{r} R(\mathbf{r}, t) n_{c}(\mathbf{r}, \mathbf{t})=-\int d \epsilon I_{12}(\epsilon, t) .
$$

Combining this with Eq. (34) leads to

$$
\frac{\partial\left(\tilde{N}+N_{c}\right)}{\partial t}=0
$$

which demonstrates that the total number of particles is indeed conserved.

\section{B. Energy conservation}

We now consider the conservation of the total energy of the system. The total energy is given by

$$
\begin{aligned}
E_{\mathrm{tot}}= & \int \frac{d \mathbf{r} d \mathbf{p}}{(2 \pi \hbar)^{3}}\left\{\frac{\mathbf{p}^{2}}{2 m}+U_{\mathrm{ext}}(\mathbf{r})+g[\tilde{n}(\mathbf{r}, t)\right. \\
& \left.\left.+2 n_{c}(\mathbf{r}, t)\right]\right\} f(\mathbf{r}, \mathbf{p}, t)+\int d \mathbf{r} \Psi^{*}(\mathbf{r}, t) \\
& \times\left[-\frac{\hbar^{2} \nabla^{2}}{2 m}+U_{\mathrm{ext}}(\mathbf{r})+\frac{g}{2} n_{c}(\mathbf{r}, t)\right] \Psi(\mathbf{r}, t) .
\end{aligned}
$$

The first term is the semiclassical expression for the total energy of the noncondensate. It contains the kinetic and external potential energy, and the Hartree-Fock mean-field interaction energy of the noncondensed cloud interacting with itself and with the condensate. We note that the selfinteraction term is reduced by a factor of two relative to the condensate term to avoid double counting this contribution.

The second term in Eq. (38) is the total energy of the condensate which contains the wave function $\Psi(\mathbf{r}, t)$ with normalization

$$
\int d \mathbf{r}|\Psi(\mathbf{r}, t)|^{2}=N_{c}(t) .
$$

It consists of the kinetic energy, the potential energy, and the mean-field energy due to the interaction of the condensate with itself. The mean-field interaction of the condensate with the noncondensate has already been included in the expression for the energy of the noncondensed cloud. We now show that this total energy is indeed conserved during the growth process.

Taking the time derivative of Eq. (38) leads to the following expression: 


$$
\begin{aligned}
\frac{\partial E_{\mathrm{tot}}}{\partial t}= & \int \frac{d \mathbf{r} d \mathbf{p}}{(2 \pi \hbar)^{3}}\left\{\frac{\mathbf{p}^{2}}{2 m}+U_{\mathrm{ext}}(\mathbf{r})+2 g[\tilde{n}(\mathbf{r}, t)\right. \\
& \left.\left.+n_{c}(\mathbf{r}, t)\right]\right\} \frac{\partial f(\mathbf{r}, \mathbf{p}, t)}{\partial t}+\int d \mathbf{r} \frac{\partial \Psi^{*}(\mathbf{r}, t)}{\partial t} \\
& \times\left\{-\frac{\hbar^{2} \boldsymbol{\nabla}^{2}}{2 m}+U_{\mathrm{ext}}(\mathbf{r})+g\left[n_{c}(\mathbf{r}, t)+2 \tilde{n}(\mathbf{r}, t)\right]\right\} \Psi(\mathbf{r}, t) \\
& +\int d \mathbf{r} \Psi^{*}(\mathbf{r}, t)\left\{-\frac{\hbar^{2} \nabla^{2}}{2 m}+U_{\mathrm{ext}}(\mathbf{r})+g\left[n_{c}(\mathbf{r}, t)\right.\right. \\
& +2 \tilde{n}(\mathbf{r}, t)]\} \frac{\partial \Psi(\mathbf{r}, t)}{\partial t} .
\end{aligned}
$$

The first term in Eq. (40) can be rewritten as

$$
\begin{aligned}
& \int \frac{d \mathbf{r} d \mathbf{p}}{(2 \pi \hbar)^{3}} E(\mathbf{r}, \mathbf{p}, t) \frac{\partial f(\mathbf{r}, \mathbf{p}, t)}{\partial t} \\
& \quad=\int \frac{d \mathbf{r} d \mathbf{p}}{(2 \pi \hbar)^{3}} E(\mathbf{r}, \mathbf{p}, t)\left(C_{12}[f]+C_{22}[f]\right) \\
& \quad=\int d \mathbf{r} E_{c}(\mathbf{r}, t) \int \frac{d \mathbf{p}}{(2 \pi \hbar)^{3}} C_{12}[f],
\end{aligned}
$$

where, to obtain this result, we have used the kinetic equation, Eq. (1), and the fact that the $C_{22}$ collision integral conserves energy.

If we again assume that the condensate grows adiabatically as atoms are fed into it from the noncondensate, the condensate wave function $\Psi(\mathbf{r}, t)$ is a solution of the instantaneous Gross-Pitaevskii (GP) equation

$$
\begin{aligned}
& \left\{-\frac{\hbar^{2} \boldsymbol{\nabla}^{2}}{2 m}+U_{\mathrm{ext}}+g\left[n_{c}(\mathbf{r}, t)+2 \tilde{n}(\mathbf{r}, t)\right]\right\} \Psi(\mathbf{r}, t) \\
& =E_{c}(t) \Psi(\mathbf{r}, t),
\end{aligned}
$$

with a time-dependent energy eigenvalue $E_{c}(t)$. For this spatially independent condensate energy, Eq. (41) reduces to

$$
\int \frac{d \mathbf{r} d \mathbf{p}}{(2 \pi \hbar)^{3}} E(\mathbf{r}, \mathbf{p}, t) \frac{\partial f(\mathbf{r}, \mathbf{p}, t)}{\partial t}=E_{c}(t) \frac{\partial \tilde{N}(t)}{\partial t} .
$$

Inserting this result and Eq. (41) into Eq. (40), the latter is easily seen to yield

$$
\frac{\partial E_{\mathrm{tot}}}{\partial t}=E_{c}(t)\left(\frac{\partial \tilde{N}}{\partial t}+\frac{\partial N_{c}}{\partial t}\right)=0
$$

due to the conservation of the total particle number. Thus the assumption of adiabaticity is sufficient to ensure that the total energy is conserved. However, one can also show the conservation of energy exactly, without assuming adiabaticity, by making use of the dissipative nonlinear Schrödinger equation in Eq. (7).

\section{THOMAS-FERMI APPROXIMATION}

The assumption that the condensate grows adiabatically implies that the dynamics of the condensate itself is being neglected, apart from its trivial time-dependent normalization. In particular, we are ignoring the possible excitation of internal collective oscillations. However, at the temperatures of interest in the growth process, these excitations are strongly damped and we expect the condensate to remain in a relatively quiescent state that is well-approximated by the quasiequilibrium solution of the GP equation. Indeed, in the experiments there is no evidence of condensate oscillations, although the thermal cloud has been observed to oscillate at twice the harmonic-oscillator frequency of the trap in some cases.

For a large number of condensate atoms, a good approximation to the equilibrium wave function is provided by the Thomas-Fermi approximation that neglects the kinetic energy in the GP equation. In this situation, the condensate density is given by

$$
n_{c}(\mathbf{r}, t)=\frac{1}{g}\left[\mu_{c}(t)-U_{\text {ext }}(\mathbf{r})-2 g \tilde{n}(\mathbf{r}, t)\right] .
$$

Of course, this expression is only valid if the right-hand side is larger than zero; otherwise, $n_{c}(\mathbf{r}, t)=0$. The last term on the right-hand side reflects the mean-field interaction of the condensate with the thermal cloud. Since the latter has a small density relative to the condensate, its effect on the spatial distribution of the condensate is small $\left(2 g \tilde{n} \ll \mu_{c}\right)$ and we therefore neglect it when determining the condensate density. By the same token, we shall neglect the mean-field interaction of the noncondensate with itself. Strictly speaking, these approximations lead to a violation of total energy conservation, but the error will be very small since the bulk of the mean-field energy, which resides within the condensate itself, is still taken into account. In principle, these contributions can be included in our treatment as shown explicitly in the Appendix. However, because these corrections are small, we have decided to neglect them in our numerical calculations.

It should be noted that the Thomas-Fermi approximation is to some extent dictated by our semiclassical treatment of the noncondensate atoms, since it avoids a potential problem associated with the placement of the condensate chemical potential $\mu_{c}$ relative to the minimum energy available to the thermal atoms, i.e., $U_{\min }=\min \left[U_{\text {ext }}+2 g\left(\tilde{n}+n_{c}\right)\right]$. For small condensate densities, it is possible that the GP eigenvalue $\mu_{c}$ lies above this minimum value that is clearly impossible if a full quantum treatment of the excited states is retained. In the Thomas-Fermi approximation, there is no such problem since the chemical potential is exactly equal to $U_{\min }$.

Given these approximations, the time-dependent condensate density profile becomes

$$
n_{c}(\mathbf{r}, t)=\frac{1}{g}\left[\mu_{c}(t)-U_{\mathrm{ext}}(\mathbf{r})\right]
$$


where the external potential is taken to be a general anisotropic harmonic confining potential, $U_{\text {ext }}(\mathbf{r})=\Sigma_{i} m \omega_{i}^{2} r_{i}^{2} / 2$. This expression for the density is again only meaningful when $U_{\text {ext }}(\mathbf{r}) \leqslant \mu_{c}(t)$. The chemical potential of the condensate is given by

$$
\mu_{c}(t)=\frac{\hbar \bar{\omega}}{2}\left[15 N_{0}(t) \frac{a}{l}\right]^{2 / 5},
$$

where $\bar{\omega}=\left(\omega_{1} \omega_{2} \omega_{3}\right)^{1 / 3}$ and $l=\sqrt{\hbar / m \bar{\omega}}$. The potential experienced by the thermal atoms is then

$$
U(\mathbf{r}, t)=\left\{\begin{array}{l}
2 \mu_{c}(t)-U_{\text {ext }}(\mathbf{r}) \quad \text { if } \quad n_{c} \neq 0 \\
U_{\text {ext }}(\mathbf{r}) \quad \text { if } \quad n_{c}=0 .
\end{array}\right.
$$

The minimum value of this potential is $\mu_{c}(t)$ and occurs on the boundary of the condensate.

Three additional important quantities can also be calculated analytically. The first two are the density of states, and the weighted density of states, i.e., $\rho(\bar{\epsilon})$ and $\rho_{\mathrm{w}}(\bar{\epsilon})$, respectively. For the former we get

$$
\begin{aligned}
\rho(\overline{\boldsymbol{\epsilon}})= & \frac{m^{3 / 2}}{\sqrt{2} \pi^{2} \hbar^{3}} \int_{\bar{U}<\bar{\epsilon}} d \mathbf{r} \sqrt{\bar{\epsilon}-\bar{U}(\mathbf{r}, t)} \\
= & \frac{2}{\pi \hbar \bar{\omega}}\left[\int_{\bar{U}<\bar{\epsilon}} d y y^{2} \theta\left(\frac{2 \mu_{c}}{\hbar \bar{\omega}}-y^{2}\right) \sqrt{\frac{2\left(\bar{\epsilon}-\mu_{c}\right)}{\hbar \bar{\omega}}+y^{2}}\right. \\
& \left.+\int_{\bar{U}<\bar{\epsilon}} d y y^{2} \theta\left(y^{2}-\frac{2 \mu_{c}}{\hbar \bar{\omega}}\right) \sqrt{\frac{2\left(\bar{\epsilon}+\mu_{c}\right)}{\hbar \bar{\omega}}-y^{2}}\right] \\
\equiv & \frac{2}{\pi \hbar \bar{\omega}}\left[I_{-}(\overline{\boldsymbol{\epsilon}})+I_{+}(\overline{\boldsymbol{\epsilon}})\right]
\end{aligned}
$$

The integrals $I_{-}(\bar{\epsilon})$ and $I_{+}(\bar{\epsilon})$ are standard, and are given by

$$
\begin{gathered}
I_{-}(\overline{\boldsymbol{\epsilon}})=\frac{u_{-}^{3} x}{4}-\frac{a_{-} u_{-} x}{8}-\left.\frac{a_{-}^{2}}{8} \ln \left(x+u_{-}\right)\right|_{x=\sqrt{\max \left\{0, a_{-}\right\}}} ^{x=\sqrt{2 \mu_{c} / \hbar \bar{\omega}}}, \\
I_{+}(\overline{\boldsymbol{\epsilon}})=-\frac{u_{+}^{3} x}{4}+\frac{a_{+} u_{+} x}{8}+\left.\frac{a_{+}^{2}}{8} \arcsin \left(\frac{x}{\sqrt{a_{+}}}\right)\right|_{x=\sqrt{2 \mu_{c} / \hbar \bar{\omega}}} ^{x=\sqrt{a_{+}}},
\end{gathered}
$$

where we have defined $a_{ \pm}=2\left(\bar{\epsilon} \pm \mu_{c}\right) / \hbar \bar{\omega}$, and $u_{ \pm}$ $=\sqrt{a_{ \pm} \bar{\mp} x^{2}}$.

To obtain an analytic expression for the weighted density of states $\rho_{\mathrm{w}}(\bar{\epsilon})$ we note that

$$
\frac{\partial \bar{U}(\mathbf{r}, t)}{\partial t}=2 \frac{\partial \mu_{c}(t)}{\partial t} \theta\left[\mu_{c}(t)-U_{\mathrm{ext}}(\mathbf{r})\right]-\frac{\partial \mu_{c}(t)}{\partial t} .
$$

We therefore find that the weighted density of states is given by

$$
\begin{aligned}
\rho_{\mathrm{w}}(\overline{\boldsymbol{\epsilon}})= & \frac{\partial \mu_{c}}{\partial t}\left[-\rho(\overline{\boldsymbol{\epsilon}})+\frac{4}{\pi \hbar \bar{\omega}} \int_{\bar{U}<\bar{\epsilon}} d y y^{2}\right. \\
& \left.\times \theta\left(\frac{2 \mu_{c}}{\hbar \bar{\omega}}-y^{2}\right) \sqrt{\frac{2\left(\overline{\boldsymbol{\epsilon}}-\mu_{c}\right)}{\hbar \bar{\omega}}+y^{2}}\right] \\
= & \frac{\partial \mu_{c}}{\partial t}\left[\frac{4}{\pi \hbar \bar{\omega}} I_{-}(\overline{\boldsymbol{\epsilon}})-\rho(\overline{\boldsymbol{\epsilon}})\right] .
\end{aligned}
$$

The third important quantity that can be calculated analytically in the Thomas-Fermi approximation arises in the ergodic projection of $C_{12}$. With the variable change in Eq. (31), and noting that $U_{\min }(t)=\mu_{c}(t)$ in the Thomas-Fermi approximation, Eq. (30) can be written as

$$
\begin{aligned}
I_{12}\left(\bar{\epsilon}_{1}\right)= & \frac{m^{3} g^{2}}{2 \pi^{3} \hbar^{7}} \int d \bar{\epsilon}_{2} \int d \overline{\boldsymbol{\epsilon}}_{3} \int d \overline{\boldsymbol{\epsilon}}_{4}\left[\delta\left(\bar{\epsilon}_{1}-\overline{\boldsymbol{\epsilon}}_{2}\right)\right. \\
& \left.-\delta\left(\bar{\epsilon}_{1}-\bar{\epsilon}_{3}\right)-\delta\left(\bar{\epsilon}_{1}-\bar{\epsilon}_{4}\right)\right]\left[\left(1+g_{2}\right) g_{3} g_{4}\right. \\
& \left.-g_{2}\left(1+g_{3}\right)\left(1+g_{4}\right)\right] \\
& \times \int_{\bar{U} \leqslant \bar{\epsilon}_{\min }} d \mathbf{r} n_{c}(\mathbf{r}, t) S\left(p_{2}, p_{3}, p_{4}\right) \delta\left(\overline{\boldsymbol{\epsilon}}_{2}-\overline{\boldsymbol{\epsilon}}_{3}-\overline{\boldsymbol{\epsilon}}_{4}\right),
\end{aligned}
$$

where $p_{i}=\sqrt{2 m\left(\bar{\epsilon}_{i}-\bar{U}\right)}$. It is apparent that the integrand is symmetric in the variables $\bar{\epsilon}_{3}$ and $\bar{\epsilon}_{4}$. We can therefore assume, without loss of generality, that $\bar{\epsilon}_{2} \geqslant \bar{\epsilon}_{3} \geqslant \bar{\epsilon}_{4}$, which also implies $p_{2} \geqslant p_{3} \geqslant p_{4}$. In this situation, $S\left(p_{2}, p_{3}, p_{4}\right)$ in Eq. (29) reduces to

$$
S\left(p_{2}, p_{3}, p_{4}\right)=\frac{1}{2}\left[1-\operatorname{sgn}\left(p_{2}-p_{3}-p_{4}\right)\right]
$$

which is nonzero and equal to 1 , if

$$
p_{2}<p_{3}+p_{4} \text {. }
$$

This restricts the spatial integration in Eq. (53) to the domain specified by this inequality. Inserting the definitions of $p_{i}$ and using the conservation of energy condition $\bar{\epsilon}_{2}=\bar{\epsilon}_{3}+\bar{\epsilon}_{4}$, Eq. (55) is equivalent to

$$
F(\bar{U}) \equiv \bar{U}^{2}-\frac{4}{3}\left(\bar{\epsilon}_{3}+\bar{\epsilon}_{4}\right) \bar{U}+\frac{4}{3} \bar{\epsilon}_{3} \bar{\epsilon}_{4}>0 .
$$

The roots of $F(\bar{U})=0$ are given by

$$
\bar{U}_{ \pm}=\frac{2}{3}\left[\left(\bar{\epsilon}_{3}+\bar{\epsilon}_{4}\right) \pm \sqrt{\bar{\epsilon}_{3}^{2}-\bar{\epsilon}_{3} \bar{\epsilon}_{4}+\bar{\epsilon}_{4}^{2}}\right],
$$

in terms of which $F(\bar{U})=\left(\bar{U}-\bar{U}_{-}\right)\left(\bar{U}+\bar{U}_{+}\right)$. The requirement $F(\bar{U})>0$ is therefore satisfied for $\bar{U}<\bar{U}_{-}$and $\bar{U}$ $>\bar{U}_{+}$. The latter condition, however, is inconsistent with the constraint $\bar{U}<\bar{\epsilon}_{4}$ for the integral in Eq. (53). Because $\bar{U}_{-}$ does satisfy $\bar{U}_{-} \leqslant \bar{\epsilon}_{4}$, the net effect of the factor 
$S\left(p_{2}, p_{3}, p_{4}\right)$ in Eq. (53) is to restrict the spatial integration domain to the domain defined by $\bar{U} \leqslant \bar{U}_{-}$, i.e.,

$$
\begin{gathered}
\int_{\bar{U} \leqslant \bar{\epsilon}_{\min }} d \mathbf{r} n_{c}(\mathbf{r}, t) S\left(p_{2}, p_{3}, p_{4}\right) \delta\left(\bar{\epsilon}_{2}-\bar{\epsilon}_{3}-\bar{\epsilon}_{4}\right) \\
=\int_{\bar{U} \leqslant \bar{U}_{-}} d \mathbf{r} n_{c}(\mathbf{r}, t) \delta\left(\overline{\boldsymbol{\epsilon}}_{2}-\overline{\boldsymbol{\epsilon}}_{3}-\overline{\boldsymbol{\epsilon}}_{4}\right) .
\end{gathered}
$$

The remaining spatial integral in Eq. (58) can be carried out analytically for the Thomas-Fermi density profile. If $\bar{U}_{-}$ $\geqslant \mu_{c}$, we have simply

$$
\int_{\bar{U} \leqslant \bar{U}_{-}} d \mathbf{r} n_{c}(\mathbf{r}, t)=N_{c}(t)
$$

On the other hand, for $0 \leqslant \bar{U}_{-} \leqslant \mu_{c}$, we have

$$
\begin{aligned}
\int_{\bar{U} \leqslant \bar{U}_{-}} d \mathbf{r} n_{c}(\mathbf{r}, t)= & N_{c}(t)\left\{\frac{5}{2}\left[1-\left(1-\frac{\bar{U}_{-}}{\mu_{c}}\right)^{3 / 2}\right]\right. \\
& \left.-\frac{3}{2}\left[1-\left(1-\frac{\bar{U}_{-}}{\mu_{c}}\right)^{5 / 2}\right]\right\} .
\end{aligned}
$$

Physically, Eqs. (59) and (60) are a consequence of the kinematical constraints for scattering into the condensate that appear in the original form of the collision integral in Eq. (30).

Finally, we indicate some implications of the assumption of adiabatic growth in the context of the Thomas-Fermi approximation. We take as an approximate solution to the dissipative nonlinear Schrödinger equation in Eq. (7) a condensate wave function of the form

$$
\Psi(\mathbf{r}, t)=\sqrt{n_{c}(\mathbf{r}, t)} e^{i \theta(\mathbf{r}, t)},
$$

where $n_{c}(\mathbf{r}, t)$ is the Thomas-Fermi density profile in Eq. (46). Inserting this wave function into Eq. (7), neglecting the kinetic-energy term as in the Thomas-Fermi approximation, and separating real and imaginary parts of the resulting equation, we obtain the relations

$$
\theta(\mathbf{r}, t)=-\frac{1}{\hbar} \int_{0}^{t} d t^{\prime} \mu_{c}\left(t^{\prime}\right),
$$

and

$$
R(\mathbf{r}, t)=-\frac{\hbar \dot{\mu}_{c}(t)}{2 g n_{c}(\mathbf{r}, t)} .
$$

The fact that the phase is spatially independent implies that the superfluid velocity $\mathbf{v}_{c}$ is zero as we have assumed in Sec. III. According to Eq. (36), Eq. (63) implies

$$
\frac{\partial N_{c}}{\partial t}=\frac{1}{g} \int d \mathbf{r} \dot{\mu}_{c}(t)
$$

where the integral is restricted to the region occupied by the condensate, i.e., $n_{c}(\mathbf{r}, t) \neq 0$. This is the same expression ob- tained by taking the time derivative of the integral of Eq. (46) over all space. We therefore see that the wave function in Eq. (61) is an internally consistent solution of the dissipative nonlinear Schrödinger equation.

\section{RESULTS}

In this section, we present the results of our calculations, which were performed for the situation corresponding to the MIT experiments [10]. These used ${ }^{23} \mathrm{Na}$ atoms confined in an axially symmetric trap with harmonic frequencies of 18.0 $\mathrm{Hz}$ and $82.3 \mathrm{~Hz}$ along and perpendicular to the symmetry axis, respectively. These values give an averaged frequency of $\bar{\omega} / 2 \pi=49.6 \mathrm{~Hz}$, which implies that $\hbar \bar{\omega} / k_{B}$ is equal to 2.4 $\mathrm{nK}$. The $s$-wave scattering length $a$ is $2.75 \mathrm{~nm}$.

To begin, we provide a few of the numerical details. We used a discretized energy mesh consisting of equally spaced points in the range $0 \leqslant \bar{\epsilon} \leqslant \bar{\epsilon}_{\max }$. The value of the temperature used in the simulations is typically of the order of $1 \mu \mathrm{K}$, which requires a maximum energy range of about $\bar{\epsilon}_{\max } \simeq 2500-3000 \hbar \bar{\omega}$ in order to ensure that $\rho(\bar{\epsilon}) g(\bar{\epsilon})$ is sufficiently small at the end of the range. In evaluating the collision integrals $I_{22}$ in Eq. (26) and $I_{12}$ in Eq. (30), the delta functions were used to perform some of the integrations analytically. The remaining integrals were then evaluated numerically using a simple trapezoidal integration scheme. The main advantage of this scheme in the case of the $I_{22}$ collision integral is that the conservation of both particle number and energy is numerically exact, which in general is not the case for higher-order integration schemes such as Simpson's rule. This conserving property is especially important in simulations of the condensate growth since a loss of either particles or energy due to numerical inaccuracy would lead to systematic errors in the final equilibrium values for various physical quantities. The situation for the $I_{12}$ collision integral is somewhat different since neither of the integrals in Eq. (35) or (36) is zero. Thus the numerical results will depend on the choice of the energy-mesh size, and one must check that the results obtained for a given simulation are insensitive to variations in this parameter. The checks we have performed indicate that errors in the final results coming from this numerical source are no larger than a few percent. Errors of this magnitude will not influence the general conclusions that we make. As a final point, we used the Euler method to propagate Eq. (32) in time. The time step was chosen to be sufficiently small, typically $0.5 \mathrm{~ms}$, to ensure the accuracy of the time evolution.

To start the simulation, we begin with an initial nonequilibrium distribution that is meant to represent the conditions immediately after the rapid evaporative cooling quench used in the experiments. Ideally, such a quench starts with an equilibrium distribution at some temperature $T$ above $T_{c}$ and excises all particles with energy above $E_{\text {cut }} \equiv k_{B} T_{\text {cut }}$. We model this by a truncated Bose distribution at the temperature $T$. Although we expect this initial distribution to represent the experimental situation reasonably well, there will no doubt be differences from the actual distributions due to the finite time taken to perform the quench, which allows some 


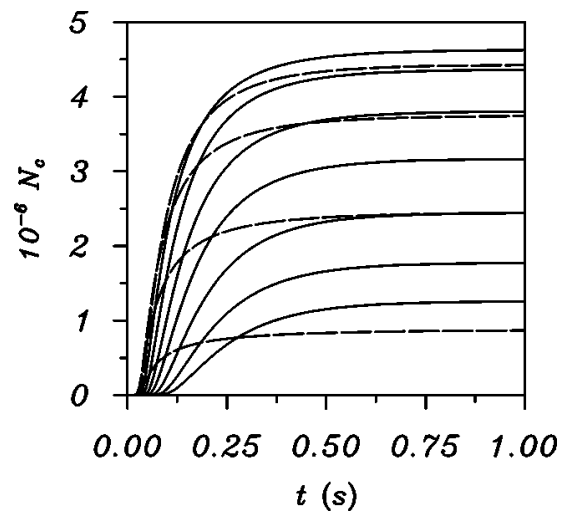

FIG. 1. Growth curves for different initial energy cutoffs. As discussed in Sec. IV, the initial conditions are defined by fixing the temperature $T=T_{c}=0.765 \mu \mathrm{K}$ and the chemical potential $\tilde{\mu}=0$ of the distribution function before it is truncated. This gives $\widetilde{N}=40$ $\times 10^{6}$ noncondensate atoms. The number of atoms initially in the condensate is chosen to be $N_{c}=214$. The solid curves in order of increasing saturation values correspond to $T_{\text {cut }} / T_{c}=5.5,5.0,4.5$, 4.0, 3.5, 3.0, and 2.5. The dashed curves in order of decreasing saturation values correspond to $T_{\text {cut }} / T_{c}=2.0,1.5,1.0$, and 0.5 .

equilibration to occur, and the possible incomplete removal of all particles in the energy range of the sweep. Since the RF field is resonant only at certain positions in the trap, atoms of a given energy must have sufficient time to reach these positions in order to suffer a spin flip and thus be ejected from the trap. If this is not the case, the distribution in energy will also have a spatial dependence. Some indication that such a nonergodic state in fact occurs is provided by the observation that the thermal cloud starts to oscillate after the quench. However, due to a lack of detailed information about the experimental initial conditions, we shall assume an idealized truncated Bose distribution as our initial condition.

To complete the specification of the initial state, we must also make a choice for the number of atoms initially in the condensate. Of course, if this number is zero, $I_{12}$ as given by Eq. (27) is zero since we have only included stimulated transitions into the condensate. In the absence of spontaneous processes, there is no possibility of condensate growth. Under the experimental conditions of interest, however, the lowest quantum state initially already has a rather large thermal occupation and stimulated processes will dominate. We therefore choose the initial condensate number to be given by the occupation of the lowest harmonic-oscillator state at the temperature of the truncated Bose distribution. This number is typically of the order of a few hundred particles. As our numerical results presented below will show, the growth curves are rather insensitive to this starting value as long as it is small compared to the final equilibrium number of condensate atoms.

In Fig. 1, we show a sequence of growth curves that illustrate the dependence on the parameter $T_{\text {cut }}$. In this set of simulations, we assume that the temperature of the equilibrium Bose distribution is equal to $T_{c}=0.765 \mu \mathrm{K}$ and its chemical potential $\tilde{\mu}$ is equal to zero. Before the cut, the gas contains $\widetilde{N}=40 \times 10^{6}$ thermal atoms and the number of con-

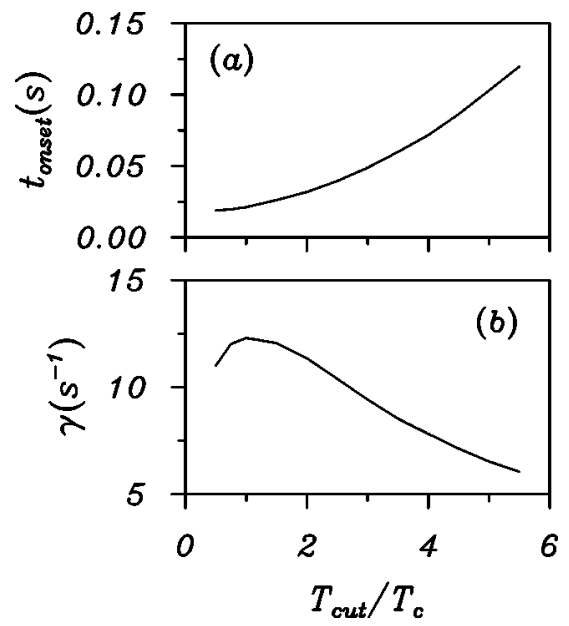

FIG. 2. The onset time (a) and relaxation rate (b) for the growth curves in Fig. 1, as determined by using the fitting function in Eq. (65).

densate atoms is given by $N_{c}=[\exp (3 \beta \hbar \bar{\omega} / 2)-1]^{-1}=214$. In a particular simulation, the total number of atoms and the average energy per atom of course depends on the depth of the energy cut. The growth curves are characterized by an initial stage of slow growth during which the truncated Bose distribution evolves into a quasiequilibrium distribution, a well-defined onset time $t_{\text {onset }}$ where a significant increase in the rate of growth occurs, and finally a relaxational stage where the condensate number approaches a final equilibrium value. As the cut is made deeper and deeper, this final number at first increases due to the decreasing total energy of the initial distribution, which results in a lower final temperature. However, at some point the final number of condensate atoms reaches a maximum and then decreases with further deepening of the cut due to the reduced total number of atoms in the initial distribution. To distinguish this behavior, the growth curves are shown as solid lines when the final number is increasing with decreasing $T_{\text {cut }}$, and conversely, by dashed lines when the final number is decreasing.

Although all the growth curves in Fig. 1 are qualitatively similar, it is clear that there are important differences in detail. For the curves with an increasing equilibrium number of condensate particles, i.e., the solid curves, both the onset time and subsequent relaxation time are seen to decrease with decreasing $T_{\text {cut }}$. However, for the curves with a decreasing equilibrium number of condensate particles, i.e., the dashed curves, the dependence of both of these times on further decreases in $T_{\text {cut }}$ is much weaker, and they appear to approach limiting values. In order to quantify this behavior, it is convenient to fit the relaxational part of the theoretical growth curves to a simple exponential relaxation

$$
N_{c}^{\mathrm{fit}}(t) \simeq N_{c}^{\mathrm{eq}}\left(1-e^{-\gamma\left(t-t_{\text {onset }}\right)}\right),
$$

where $N_{c}^{\mathrm{eq}}, \gamma$, and $t_{\text {onset }}$ are fitting parameters. This functional form is found to provide a very good fit to this part of the theoretical curves. Fig. 2 summarizes the results for the onset time $t_{\text {onset }}$, and exponential relaxation rate $\gamma$ for the particular simulations presented in Fig. 1. The onset time 


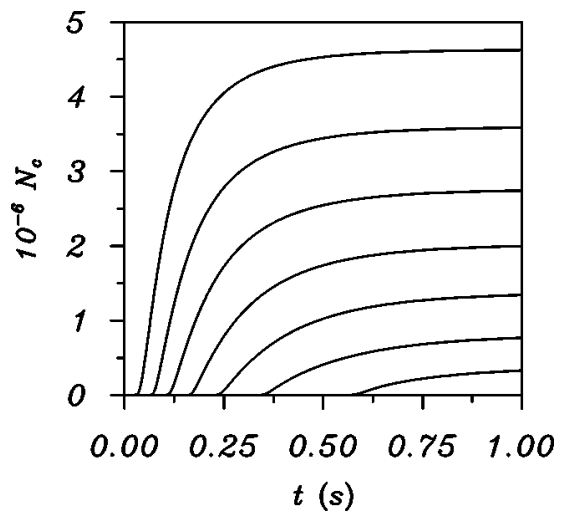

FIG. 3. Growth curves for different initial temperatures $T$. In order of decreasing equilibrium number of condensate atoms, $T / T_{c}=1,1.05,1.1,1.15,1.2,1.25$, and 1.3. The initial conditions are defined by fixing the number of noncondensed particles to $\widetilde{N}$ $=40 \times 10^{6}$, and the number of condensed atoms to $N_{c}=214$, as in Fig. 1. The cutoff is now kept fixed at $T_{\text {cut }} / T_{c}=2.5$. The chemical potential is less than zero, and adjusted to keep the number of noncondensed particles fixed.

decreases from about $100 \mathrm{~ms}$ to $20 \mathrm{~ms}$ as $T_{\text {cut }} / T_{c}$ is reduced from 5 to 0.5 . At the same time, the relaxation rate increases from about $6 \mathrm{~s}^{-1}$ to $12 \mathrm{~s}^{-1}$.

We have also looked at the dependence of the growth curves on the other parameters that appear in the theory. In Fig. 3, we show the growth curves for a range of initial temperatures. Prior to the quench, these initial temperatures are larger than $T_{c}$, and in each case the chemical potential is adjusted to provide again a total of $40 \times 10^{6}$ atoms in the thermal cloud. The energy cut and initial number of condensate atoms were taken to be $T_{\text {cut }} / T_{c}=2.5$ and $N_{c}(0)=214$, respectively, and were the same for all the runs. Not surprisingly, we find that the final equilibrium condensate number decreases with increasing initial temperature as a result of the larger average energy per atom. This of course also leads to a higher final equilibrium temperature. However, what is somewhat unexpected is the very rapid increase of the onset time as the initial temperature is increased. In Fig. 4(a), we show that a $30 \%$ variation in $T / T_{c}$ gives rise to more than a tenfold variation in $t_{\mathrm{onset}}$, and that these values are typically much larger than those found using an initial temperature of $T=T_{c}$. In addition, Fig. 4(b) shows that the relaxation rate tends to decrease with increasing $T / T_{c}$ and is comparable to the values given in Fig. 2.

In Fig. 5, we show the variation of the growth curves with the initial number of condensate atoms. In this case, the initial nonequilibrium distribution is held fixed, corresponding to a Bose distribution with $\widetilde{N}=40 \times 10^{6}, T=T_{c}$, and $T_{\text {cut }} / T_{c}=2.5$. The growth curve is rather insensitive to the condensate number in the range $10^{2}<N_{c}<10^{4}$, but then shows a much stronger dependence in the range $10^{4}<N_{c}$ $<10^{6}$. At the higher end of this range, the initial number is already visible on the graph and by $N_{c}=10^{6}$ there is no longer a meaningful onset time. This would correspond to a situation in which a significant condensate fraction has al-

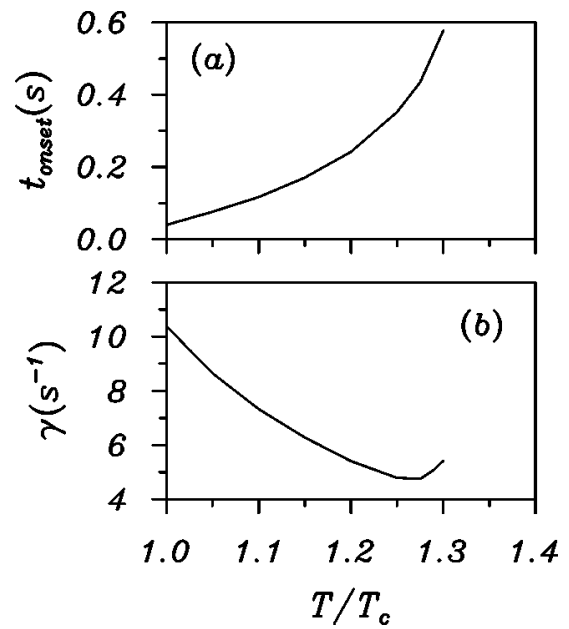

FIG. 4. The onset time (a) and relaxation rate (b) for the growth curves in Fig. 3, using the same fitting procedure as in Fig. 2.

ready formed by the time the quench is completed. This kind of behavior is indeed also seen experimentally under certain conditions.

In order to explain some of these results, it is necessary to examine the time evolution of the distribution function $g(\bar{\epsilon}, t)$. In Fig. 6, we show $\ln (g)$ vs $\bar{\epsilon}$ for various times after the quench. At early times the distribution function is equilibrated by the scattering of thermal atoms into states above the $E_{\text {cut }}$ that are initially depleted. To conserve energy, the mean energy of the atoms below $E_{\text {cut }}$ must decrease. In fact, the population of the low-energy states increases significantly before the onset of rapid condensate growth. This is shown in Fig. 7 where $g(\bar{\epsilon}, t)$ is plotted as a function of time for some specific energy values. We see that $g(\bar{\epsilon}, t)$ at first increases rapidly, reaches a maximum at a time very close to the onset time and then relaxes toward its final equilibrium value of $\left(e^{\beta_{\mathrm{eq}} \bar{\epsilon}}-1\right)^{-1}$. This behavior is typical of all situations in which the growth of a condensate is observed. This strong correlation of the peak position in Fig. 7 with the onset time suggests that condensate formation is triggered by

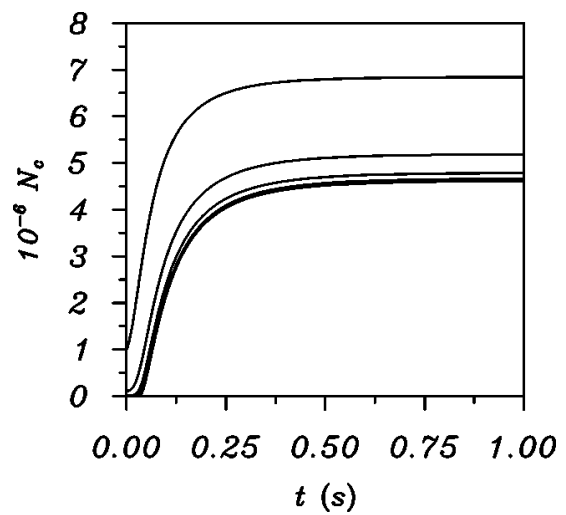

FIG. 5. Growth curves for different initial number of condensed particles. The other parameters defining the initial conditions are the same as in Fig. 1, and the cutoff is held fixed at $T_{\text {cut }} / T_{c}=2.5$. In order of increasing saturation values, the curves correspond to $N_{c}(0)=10^{2}, 10^{3}, 10^{4}, 10^{5}$, and $10^{6}$. 


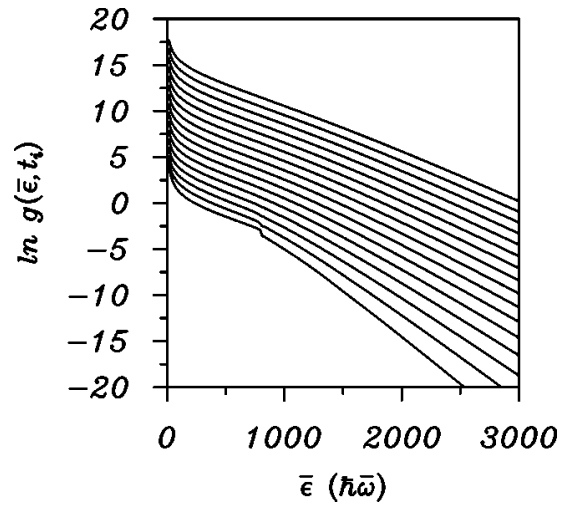

FIG. 6. Plot of the logarithm of the distribution function $g(\epsilon, t)$ for the curve with $T_{\text {cut }} / T_{c}=2.5$ from Fig. 1, at time intervals $\Delta t$ $=0.02 \mathrm{~s}$, starting from $t=0.02 \mathrm{~s}$. Each curve is shifted up by one unit with respect to the previous one for clarity.

an enhanced low-energy population. Before the onset time, we find numerically that $g(\bar{\epsilon})$ behaves approximately as $(\bar{\epsilon})^{-1.63}$, which is a stronger singularity than that exhibited by an equilibrium Bose distribution with zero chemical potential, and agrees within our numerical accuracy with the $(\bar{\epsilon})^{-5 / 3}$ dependence predicted by Svistunov [23]. Regardless of the precise exponent, it seems that a "supercritical", behavior of the distribution function is a precursor to condensate formation [24].

A useful way to characterize the time evolution of $g(\bar{\epsilon}, t)$ is to express it locally as a Bose distribution

$$
g(\bar{\epsilon}, t)=\frac{1}{\exp (\beta \bar{\epsilon}-\tilde{\mu})-1},
$$

where the two parameters $\beta$ and $\tilde{\mu}$ are defined by fitting this expression to the value of the distribution function and its energy derivative. Although the parameters are treated locally as constants in this procedure, they nevertheless depend parametrically on the energy variable $\bar{\epsilon}$. The local temperature and chemical potential parameters defined in this way

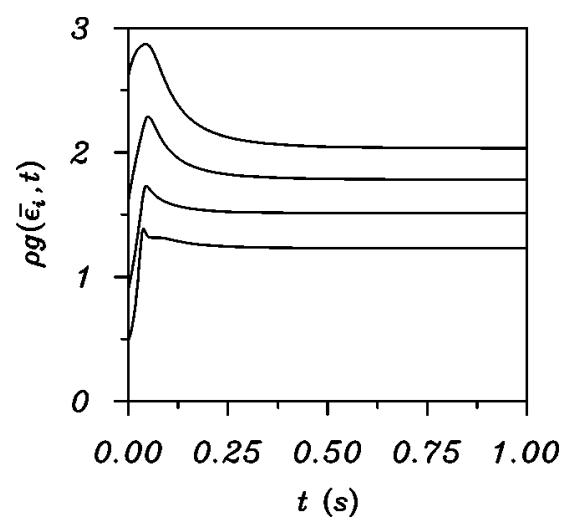

FIG. 7. Plot of the product of the density of states and the distribution function at energies of $30,60,120$, and $240 \hbar \bar{\omega}$, for the curve with $T_{\text {cut }} / T_{c}=2.5$ in Fig. 1 . The peaks occur in the vicinity of the onset time $t_{\text {onset }}$.

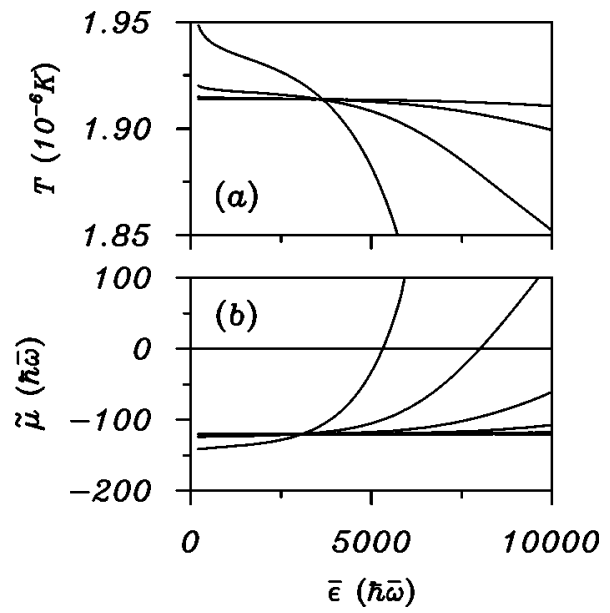

FIG. 8. Equilibration of the local temperature and chemical potential for a situation in which the final equilibrium temperature of the gas is above the critical temperature. Panel (a) gives the local temperature as a function of energy for a sequence of times during the equilibration process. In equilibrium, both the temperature and chemical potential are independent of energy. Panel (b) gives the corresponding variation of the local chemical potential. The initial conditions before the distribution is truncated are defined by a temperature $T=2 \mu \mathrm{K}$, and a chemical potential $\tilde{\mu}=-200$. The cutoff is at $T_{\text {cut }} / T_{c}=2.5$.

are shown in Fig. 8(a) and 8(b) at time intervals of $0.05 \mathrm{~s}$ for a situation in which the quenched thermal cloud equilibrates to a final temperature above $T_{c}$. Both parameters are seen to be strongly energy-dependent at early times but evolve toward energy-independent values by the end of the simulation. The negative equilibrium value of the chemical potential corresponds to an uncondensed thermal cloud at a temperature of about $1.92 \mu \mathrm{K}$.

A situation in which the quench leads to the formation of a condensate is illustrated in Figs. 9(a) and 9(b). The parameters are plotted at $0.25 \mathrm{~s}$ intervals during the relaxational stage of the growth curve beyond the onset time. At low

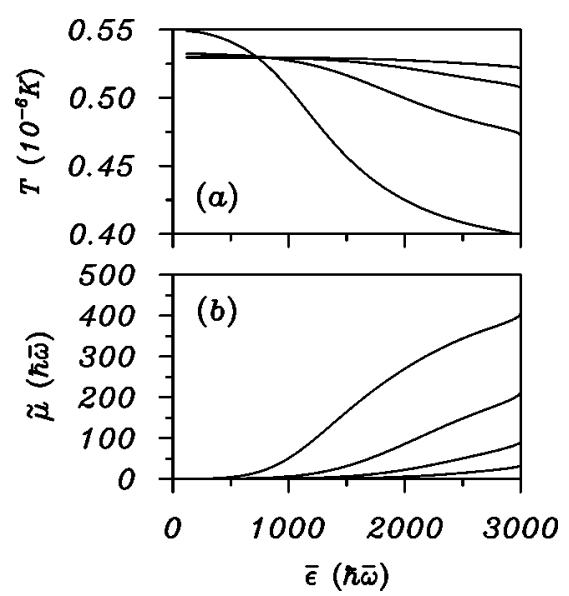

FIG. 9. As in Fig. 8, but for a situation in which the final equilibrium temperature is below the critical temperature. The equilibration of the local temperature and chemical potential corresponds to the $T_{\text {cut }} / T_{c}=2.5$ growth curve in Fig. 1 . 


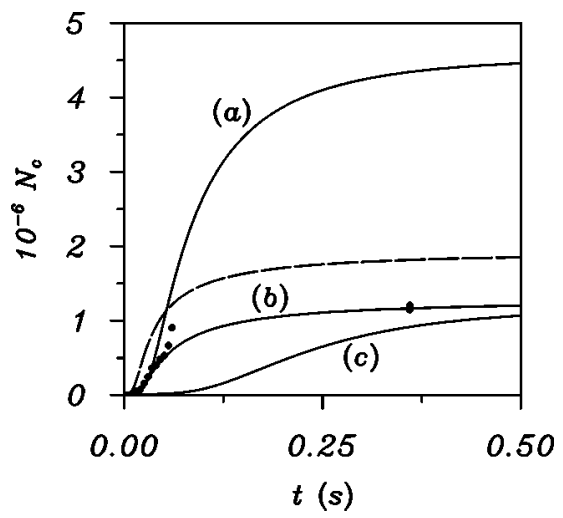

FIG. 10. Theoretical growth curves for the initial conditions of Fig. 1, but with $N_{c}(0)=10^{4}$ and for various energy cuts: (a) $T_{\text {cut }} / T_{c}=1.9$, (b) $T_{\text {cut }} / T_{c}=0.6$, (c) $T_{\text {cut }} / T_{c}=5.7$. The experimental points are taken from Fig. 4 of Ref. [10]. The dashed line shows the theoretical growth curve for the conditions of case (b) but with mean-field interactions turned off.

energies, the local temperature lies above the final equilibrium value that reflects the higher temperature of the initial Bose distribution. However at higher energies, the local temperature is lower than the final temperature since the gas in this energy range is effectively colder as a result of the quench. Figure 9(b) shows the corresponding variation of the chemical potential. As a result of the formation of the condensate, the chemical potential at low energies is pinned to zero and then increases at higher energies. The deviations of both the local temperature and chemical potential from their final equilibrium values are seen to relax to zero on a time scale that is comparable with the relaxational stage of the condensate growth. This relaxation rate can therefore be attributed to the relatively slow equilibration of the local temperature and chemical potential of the thermal cloud.

We finally turn to a comparison with experiment. This is shown in Fig. 10 for the particular case in which the starting number of noncondensed atoms is $40 \times 10^{6}$, as in the simulations discussed above, but with the initial number of condensate atoms set to $N_{c}(0)=10^{4}$. In the particular experimental run starting with this total number of atoms before the RF quench, the condensate number is found to relax to a final number of $1.2 \times 10^{6}$ atoms. According to Fig. 1, there are two values of $T_{\text {cut }}$ that will lead to this final number of condensate atoms, $T_{\text {cut }} / T_{c}=0.6$ and $T_{\text {cut }} / T_{c}=5.7$. The results for the deeper cut of $T_{\text {cut }} / T_{c}=0.6$ are shown as curve (b) and are seen to be in very good agreement with the experimental results. However, we cannot claim good agreement overall since the total number of atoms after the quench is only $2.5 \times 10^{6}$ as compared to the experimental number of about $16.0 \times 10^{6}$ atoms. For the shallower cut of $T_{\text {cut }} / T_{c}$ $=5.7$ shown as curve (c), the agreement between the theoretical and experimental growth curves is clearly worse in that the theoretical growth rate is too small. Moreover, the total number of atoms remaining in the trap is $37.5 \times 10^{6}$ that is too large by roughly a factor of 2 . Alternatively, one can choose a cut that reproduces the final number of atoms in the trap. In our simulations, this requires a cut of $T_{\text {cut }} / T_{c}=1.9$. Although the initial growth rate agrees with experiment in

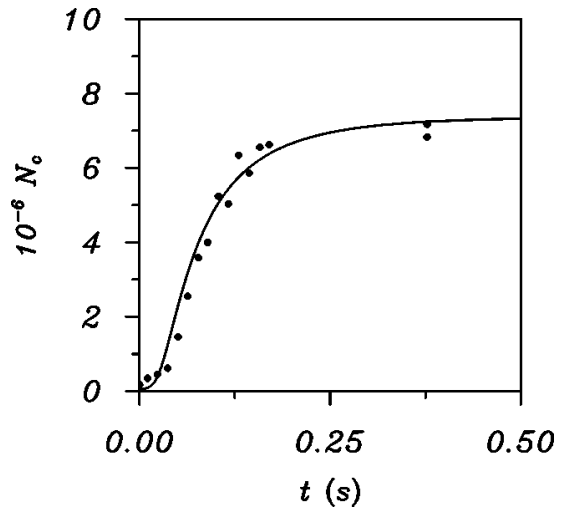

FIG. 11. Theoretical growth curve for initial conditions given by $\tilde{N}(0)=60 \times 10^{6}, \quad T=T_{c}=0.876 \mu \mathrm{K}, \quad T_{\text {cut }} / T_{c}=2.5, \quad$ and $N_{c}(0)$ $=50 \times 10^{4}$. The experimental points are taken from Fig. 3 of Ref. [10].

this case, the final equilibrium number of condensate atoms is $4.5 \times 10^{6}$, which is too large by almost a factor of 4 . This final number could be improved by elevating the starting temperature (recall that these simulations used $T=T_{c}$ $=0.765 \mu \mathrm{K}$ ), however as Fig. 3 shows, achieving a fourfold reduction in the equilibrium condensate number would increase the onset time well beyond the experimental value. It therefore appears that the present simulations cannot reproduce all aspects of the experiments simultaneously.

Figure 10 also shows a theoretical growth curve for the same initial conditions as for curve (b), but with mean-field interactions between the condensate and thermal cloud turned off. To elaborate, the potential acting on the thermal cloud is simply the time-independent trapping potential, and the condensate is taken to have essentially a delta function spatial distribution at zero energy. In this case, the integral of $n_{c}(\mathbf{r}, \mathbf{t})$ in Eq. (30) is replaced by $N_{c}(t)$. It can be seen that the qualitative behavior is very similar to the fully interacting simulation, but that the equilibrium number of condensate atoms is increased considerably, as expected.

Figure 11 provides a comparison with another set of experimental results. In this case the initial number of atoms before the quench is not known and was therefore taken to be $60 \times 10^{6}$ in order to optimize agreement with experiment. Furthermore, the energy cut was chosen as $T_{\text {cut }} / T_{c}=2.5$. This leads to a final number of $7.3 \times 10^{6}$ condensate atoms in the trap, which is approximately the same number as found in the experiment, $N_{c}=7.2 \times 10^{6}$. Although this simulation achieves good agreement between theory and experiment for the condensate growth curve, there are too many unknown variables, including the final number of atoms in the trap, to know whether or not theory is reproducing experiment. For this reason, the results in Fig. 11 should simply be viewed as a possible fit to the experimental data.

\section{DISCUSSION AND OUTLOOK}

Our main objective has been to obtain a realistic description of condensate growth that takes into account the effects of mean-field interactions. Within the ergodic approximation for the noncondensed atoms, and the adiabatic approxima- 
tion for the condensate, the kinetic equation we obtain is given by Eq. (19), and we have used this equation to perform simulations of condensate growth. In agreement with earlier work $[18,21]$, we find that the growth curves have a welldefined onset time, after which an exponential relaxation toward equilibrium takes place. Detailed comparison with the results reported in Ref. [10] shows that certain parameters can be tuned in order to achieve agreement with the experimental growth curves. However, it seems impossible with the present simulations to reproduce the overall equilibrium state of the trapped gas.

If we attribute the existing discrepancies to theory, we must at some point reexamine the two major assumptions made in this work, namely the adiabatic growth of the condensate and the ergodic evolution of the thermal cloud. The adiabatic assumption neglects the dynamics of the condensate, specifically the possibility that collective oscillations are excited during the growth process. Whether or not this has any important effect on the rate at which atoms are exchanged between the condensate and thermal cloud in not known and should be investigated. In the same vein, oscillations of the thermal cloud seen in the experiments clearly indicate the nonergodic state of the gas that in principle might be important in determining the time scale of equilibration. However, to answer this question requires a solution of the full quantum Boltzmann equation that seems out of reach at the moment. One cannot of course discount the possibility that there are uncertainties in the experimental results themselves. Further experimental work is needed to confirm the earlier results and to explore in more detail the dependences on various parameters such as the initial temperature of the cloud and the depth of the RF cut.

After completion of this work, a preprint by Davis, Gardiner, and Ballagh appeared [25] that is a continuation of a series of papers by Gardiner et al. It also addresses the issue of mean-field interactions as affecting the density of states, and improves on the authors' earlier work by giving a more realistic description of the RF quench used in the experiments. Thus, although there are differences in methodology, the physical basis of their work and the approximations they make are essentially equivalent to ours. As confirmation of this equivalence, their calculations of condensate growth performed for the initial conditions of Figs. 10 and 11 yield results that are in quantitative agreement with ours. The situation considered in Fig. 10 is optimal from a theoretical point of view since the experimental conditions are best known in this case. Yet both sets of calculations are unable to reproduce the experimental results in every detail.

One of the differences between their work and ours concerns the way that the condensate is treated. In our formulation, the condensate is isolated explicitly as the macroscopically occupied quantum state, and the remaining excited states making up the thermal cloud are treated semiclassically. As a result of this formulation, we have two kinds of collision integrals, one for thermal atoms scattering among each other and a second for collisions of thermal atoms with the condensate. In the formulation of Davis, Gardiner, and Ballagh on the other hand, all states including the condensate are treated equivalently and thus only a single collision inte- gral enters. As a result, the effective collision cross section involving the condensate does not depend on time as it does in our formulation. A second apparent difference has to do with the term involving the weighted density of states $\rho_{\mathrm{w}}$ in Eq. (19). This term arises as a consequence of the time dependence of the mean-field interaction. Although Davis, Gardiner, and Ballagh also deal with a time-dependent density of states, the second term on the right-hand side of Eq. (15) does not appear explicitly in their kinetic equation. However, they account for this term by dividing phase space into energy bins having widths that are a function of time. A final difference involves the use of the Bogoliubov excitation spectrum in the calculation of their density of states, instead of the Hartree-Fock dispersion used here. We do not expect this to affect the condensate growth curves significantly. However, if quasiparticle excitations are invoked, one should in principle also use these states to calculate the collision integrals [14]. It is not known at present what effect this might have on the collision rates for the low-lying energy levels.

Finally, we note that the ergodic treatment of the Boltzmann equation is a powerful, albeit approximate, method that would allow the study of nonequilibrium processes in other situations as well. Some future applications might include the nonequilibrium dynamics of fermion-fermion and boson-fermion mixtures. Thus far, the problem of evaporative cooling in these systems has been studied using a simplified procedure whereby the distribution function is assumed to be given by a cutoff equilibrium distribution function [26]. A cooling trajectory in phase space is then generated by solving for the temperature, chemical potential and cutoff energy at each successive time step. The accuracy of this approach could be checked by solving for the entire distribution function following the methods used here. Another interesting application would be to study a nonequilibrium steady-state situation in which atoms are continuously fed into the trapping potential while simultaneously being removed by an RF cut [27]. This would be relevant to the study of steady-state atom lasers.

\section{ACKNOWLEDGMENTS}

E.Z. acknowledges the FOM for its financial support during a sabbatical visit to the University of Utrecht, as well as support from the Natural Sciences and Engineering Research Council of Canada. We would like to acknowledge useful discussions with A. Griffin and J. Williams, and with C. W. Gardiner regarding the work in Ref. [25].

\section{APPENDIX: EVALUATION OF THE WEIGHTED DENSITY OF STATES.}

In this appendix, we summarize the steps needed to include in our calculations the effect of the mean-field interactions arising from the noncondensed cloud itself. Referring to Eq. (16), we see that we must evaluate $\partial U(\mathbf{r}, t) / \partial t$. This quantity is given by

$$
\frac{\partial U(\mathbf{r}, t)}{\partial t}=2 g\left(\frac{\partial \tilde{n}(\mathbf{r}, t)}{\partial t}+\frac{\partial n_{c}(\mathbf{r}, t)}{\partial t}\right) .
$$


The time derivative of $\tilde{n}(\mathbf{r}, t)$ can be expressed as

$$
\begin{aligned}
\frac{\partial \tilde{n}(\mathbf{r}, t)}{\partial t} & =\frac{\partial}{\partial t} \int \frac{d^{3} p}{(2 \pi \hbar)^{3}} \int d \epsilon \delta(\epsilon-E(\mathbf{r}, \mathbf{p}, t)) g(\epsilon, t) \\
& =\int d \epsilon \rho(\mathbf{r}, \epsilon, t)\left[\frac{\partial U(\mathbf{r}, t)}{\partial t} \frac{\partial g(\boldsymbol{\epsilon}, t)}{\partial \epsilon}+\frac{\partial g(\epsilon, t)}{\partial t}\right] \\
& =I(\mathbf{r}, t) \frac{\partial U(\mathbf{r}, t)}{\partial t}+\int d \epsilon \rho(\mathbf{r}, \epsilon, t) \frac{\partial g(\epsilon, t)}{\partial t},
\end{aligned}
$$

where we have defined

$$
I(\mathbf{r}, t) \equiv \int d \epsilon \rho(\mathbf{r}, \epsilon, t) \frac{\partial g(\epsilon, t)}{\partial \epsilon} .
$$

Substituting Eq. (A1) into Eq. (A2), the latter can be rearranged to provide an expression for the time rate of change of $\tilde{n}(\mathbf{r}, t)$ in terms of the time rate of change of the condensate density $n_{c}(\mathbf{r}, t)$ and the distribution function $g(\epsilon)$. We find

$$
\begin{aligned}
\frac{\partial \tilde{n}(\mathbf{r}, t)}{\partial t}= & \frac{2 g I(\mathbf{r}, t)}{1-2 g I(\mathbf{r}, t)} \frac{\partial n_{c}(\mathbf{r}, t)}{\partial t} \\
& +\int d \epsilon \frac{\rho(\mathbf{r}, \epsilon, t)}{1-2 g I(\mathbf{r}, t)} \frac{\partial g(\epsilon, t)}{\partial t} .
\end{aligned}
$$

Inserting this result into Eq. (A1), we have

$$
\begin{aligned}
\frac{\partial U(\mathbf{r}, t)}{\partial t}= & -\frac{2 g}{1-2 g I(\mathbf{r}, t)} \frac{\partial n_{c}(\mathbf{r}, t)}{\partial N_{c}} \frac{\partial \tilde{N}}{\partial t} \\
& +\frac{2 g}{1-2 g I(\mathbf{r}, t)} \int d \epsilon \rho(\mathbf{r}, \epsilon, t) \frac{\partial g(\epsilon, t)}{\partial t} .
\end{aligned}
$$

We have here made use of the fact that $n_{c}(\mathbf{r}, t)$ depends on time parametrically through $N_{c}(t)$, so that

$$
\frac{\partial n_{c}(\mathbf{r}, t)}{\partial t}=\frac{\partial n_{c}(\mathbf{r}, t)}{\partial N_{c}} \frac{\partial N_{c}}{\partial t}=-\frac{\partial n_{c}(\mathbf{r}, t)}{\partial N_{c}} \frac{\partial \tilde{N}}{\partial t} .
$$

Thus, the weighted density of states becomes

$$
\begin{aligned}
\rho_{\mathrm{w}}(\epsilon, t)= & d^{3} r \rho(\mathbf{r}, \epsilon, t) \frac{\partial U(\mathbf{r}, t)}{\partial t} \\
= & -\int d^{3} r \rho(\mathbf{r}, \epsilon, t)\left(\frac{2 g}{1-2 g I(\mathbf{r}, t)} \frac{\partial n_{c}(\mathbf{r}, t)}{\partial N_{c}}\right) \frac{\partial \widetilde{N}}{\partial} t \\
& +\int d^{3} r \rho(\mathbf{r}, \epsilon, t) \int d \epsilon^{\prime} \frac{2 g \rho\left(\mathbf{r}, \epsilon^{\prime}, t\right)}{1-2 g I(\mathbf{r}, t)} \frac{\partial g\left(\epsilon^{\prime}, t\right)}{\partial t} \\
\equiv & A(\epsilon, t) \frac{\partial \tilde{N}}{\partial t}+\int d \epsilon^{\prime} B\left(\epsilon, \epsilon^{\prime}, t\right) \frac{\partial g\left(\epsilon^{\prime}, t\right)}{\partial t},
\end{aligned}
$$

where

$$
A(\epsilon, t) \equiv-\int d^{3} r \rho(\mathbf{r}, \epsilon, t)\left(\frac{2 g}{1-2 g I(\mathbf{r}, t)} \frac{\partial n_{c}(\mathbf{r}, t)}{\partial N_{c}}\right),
$$

and

$$
B\left(\epsilon, \epsilon^{\prime}, t\right) \equiv 2 g \int d^{3} r \frac{\rho(\mathbf{r}, \epsilon, t) \rho\left(\mathbf{r}, \epsilon^{\prime}, t\right)}{1-2 g I(\mathbf{r}, t)} .
$$

We recover the expression for $\rho_{\mathrm{w}}(\epsilon, t)$ given in Eq. (52) by setting the kernel $B$ equal to zero and neglecting $I$ in the expression for $A$. It can be seen that including the mean field of the noncondensate complicates the calculations considerably, but all quantities can in principle be calculated explicitly if these refinements are desired. However, as discussed in Sec. V, we do not expect these effects to be quantitatively important.
[1] M. H. Anderson, J. R. Ensher, M. R. Matthews, C. E. Wieman, and E. A. Cornell, Science 269, 198 (1995).

[2] C. C. Bradley, C. A. Sackett, J. J. Tollett, and R. G. Hulet, Phys. Rev. Lett. 75, 1687 (1995); C. C. Bradley, C. A. Sackett, and R. G. Hulet, ibid. 78, 985 (1997).

[3] K. B. Davis, M.-O. Mewes, M. R. Andrews, N. J. van Druten, D. S. Durfee, D. M. Kurn, and W. Ketterle, Phys. Rev. Lett. 75, 3969 (1995).

[4] F. Dalfovo, S. Giorgini, L. P. Pitaevskii, and S. Stringari, Rev. Mod. Phys. 71, 463 (1999).

[5] E. Levich and V. Yakhot, Phys. Rev. B 15, 243 (1977); J. Phys. A 11, 2237 (1978).

[6] D. W. Snoke and J. P. Wolfe, Phys. Rev. B 39, 4030 (1989).

[7] H. T. C. Stoof, Phys. Rev. Lett. 66, 3148 (1991); Yu. M. Kagan, B. V. Svistunov, and G. V. Shlyapnikov, Sov. Phys. JETP 75, 387 (1992); H. T. C. Stoof, Phys. Rev. A 49, 3824 (1994); D. V. Semikoz and I. I. Tkachev, Phys. Rev. Lett. 74, 3093 (1995).

[8] P. C. Hendry, N. S. Lawson, R. A. M. Lee, P. V. E. McClin- tock, and C. H. D. Williams, Nature (London) 368, 315 (1994).

[9] T. W. B. Kibble, J. Phys. A 9, 1387 (1976); A. H. Guth, Phys. Rev. D 23, 347 (1981); A. D. Linde, Phys. Lett. 108B, 389 (1982); W. H. Zurek, Nature (London) 317, 505 (1985).

[10] H.-J. Miesner, D. M. Stamper-Kurn, M. R. Andrews, D. S. Durfee, S. Inouye, and W. Ketterle, Science 279, 1005 (1998).

[11] L. Mandel and E. Wolf, Optical Coherence and Quantum Optics (Cambridge, New York, 1995).

[12] C. W. Gardiner and P. Zoller, Phys. Rev. A 55, 2902 (1997).

[13] H. T. C. Stoof, Phys. Rev. Lett. 78, 768 (1997); J. Low Temp. Phys. 114, 11 (1999).

[14] T. R. Kirkpatrick and J. R. Dorfmann, J. Low Temp. Phys. 58, 301 (1985); 58, 399 (1985).

[15] N. P. Proukakis and K. Burnett, J. Res. Natl. Inst. Stand. Technol. 101, 457 (1996); N. P. Proukakis, K. Burnett, and H. T. C. Stoof, Phys. Rev. A 57, 1230 (1998).

[16] R. Walser, J. Williams, J. Cooper, and M. Holland, Phys. Rev. A 59, 3878 (1999). 
[17] E. Zaremba, T. Nikuni, and A. Griffin, J. Low Temp. Phys. 116, 277 (1999).

[18] C. W. Gardiner, P. Zoller, R. J. Ballagh, and M. J. Davis, Phys. Rev. Lett. 79, 1793 (1997); C. W. Gardiner, M. D. Lee, R. J. Ballagh, M. J. Davis, and P. Zoller, ibid. 81, 5266 (1998).

[19] U. Eckern, J. Low Temp. Phys. 54, 333 (1984).

[20] O. J. Luiten, M. W. Reynolds, and J. T. M. Walraven, Phys. Rev. A 53, 381 (1996).

[21] M. Holland, J. Williams, and J. Cooper, Phys. Rev. A 55, 3670 (1997).

[22] D. Jaksch, C. W. Gardiner, and P. Zoller, Phys. Rev. A 56, 575
(1997).

[23] B. V. Svistunov, J. Mosc. Phys. Soc. 1, 373 (1991).

[24] See also C. A. Sackett, J. M. Gerton, M. Welling, and R. G. Hulet, Phys. Rev. Lett. 82, 876 (1999).

[25] M. J. Davis, C. W. Gardiner, and R. J. Ballagh, e-print cond-mat/9912439.

[26] M. J. Holland, B. DeMarco, and D. S. Jin, Phys. Rev. A 61, 053610 (2000).

[27] J. Williams, R. Walser, C. Wieman, J. Cooper, and M. Holland, Phys. Rev. A 57, 2030 (1999). 